\title{
EchoGéo
}

$50 \mid 2019$

Varia

\section{Risques liés aux écoulements torrentiels et leur gestion à Chosica (Lima, Pérou)}

Évolution récente entre 2012 et 2017

Alexis Ricardo Mejía Uquiche et Josyane Ronchail

\section{OpenEdition}

Journals

Édition électronique

URL : https://journals.openedition.org/echogeo/18392

DOI : $10.4000 /$ echogeo.18392

ISSN : 1963-1197

Éditeur

Pôle de recherche pour l'organisation et la diffusion de l'information géographique (CNRS UMR 8586)

Référence électronique

Alexis Ricardo Mejía Uquiche et Josyane Ronchail, « Risques liés aux écoulements torrentiels et leur gestion à Chosica (Lima, Pérou) », EchoGéo [En ligne], 50 | 2019, mis en ligne le 31 décembre 2019, consulté le 21 septembre 2021. URL : http://journals.openedition.org/echogeo/18392 ; DOI : https:// doi.org/10.4000/echogeo.18392

Ce document a été généré automatiquement le 21 septembre 2021.

EchoGéo est mis à disposition selon les termes de la licence Creative Commons Attribution - Pas d'Utilisation Commerciale - Pas de Modification 4.0 International (CC BY-NC-ND) 


\title{
Risques liés aux écoulements torrentiels et leur gestion à Chosica (Lima, Pérou)
}

\author{
Évolution récente entre 2012 et 2017
}

Alexis Ricardo Mejía Uquiche et Josyane Ronchail

Les auteurs tiennent à remercier les représentants des ministères, des administrations locales, des associations et des ONG qui les ont aidés tout au long de leur démarche. Ils remercient également les habitants des ravins de Chosica pour leurs témoignages et leur accueil chaleureux.

\section{Introduction}

1 L'agglomération de Lima (environ 12 millions d'habitants) est vulnérable compte tenu de son exposition à de nombreux aléas susceptibles d'y causer des dommages importants (séismes, tsunami, inondation, glissements de terrain) et des difficultés qu'ont les autorités et les populations à les anticiper, y faire face et récupérer après leur survenue (D’Ercole et al., 2009). Le district de Lurigancho-Chosica (250 000 hab. environ), dans la banlieue de Lima, est situé au bord du fleuve Rimac, à une altitude comprise entre 800 et $2200 \mathrm{~m}$ dans la Cordillère occidentale des Andes (illustration 1). De nombreux ravins, la plupart du temps inactifs à cause de l'aridité de la région, entaillent des versants dénudés, aux pentes fortes $\left(20^{\circ}\right.$ à $\left.40^{\circ}\right)$ et composés de roches intrusives très altérées. Des laves torrentielles, écoulements boueux et rocailleux très rapides opérant dans des chenaux très en pentes (Flageollet 1988 ; Hungr et al., 2001 ; Perrin et al., 2000) se produisent dans ces ravins tous les 2 à 5 ans.

Elles occasionnent des dégâts considérables car les bassins versants sont très peuplés (illustration 2). On estime que $36 \%$ de la population de la ville de Chosica (i.e. 21000 personnes) réside dans des zones susceptibles d'être submergées par les laves torrentielles (INEI, 2007). En effet, depuis les années 1945-50 les sites peu dangereux et initialement urbanisés ont été largement saturés par l'arrivée de populations provenant de régions andines pauvres et la croissance urbaine s'est traduite par 
l'urbanisation de pentes peu ou pas constructibles au regard des normes de sécurité ou ne présentant pas d'enjeux fonciers (Thouret et D'Ercole, 1994). De plus la croissance démographique est très importante avec un taux d'accroissement de la population constante de 3,8 \% de 1981 à 1993 et de 1993 à 2007 (INEI 1981, 1993, 2007). Du fait du développement économique important de cette région, il est probable que la population continuera à augmenter. En effet, la vallée du Rimac est stratégique car elle pourvoit Lima en eau et relie la métropole aux régions de la Sierra du Pérou par «l'Autoroute Centrale » et une voie ferrée (Abad et al., 2009) (illustrations 1 et 2).

3 L'épisode d'écoulements torrentiels du 9 mars 1987 a été catastrophique (100 décès et 1052 logements détruits). Depuis, des mesures destinées à réduire le risque d'inondation dans le district ont été progressivement mises en place et se développent au fil du temps et des événements (Abad, 2009). C'est ainsi que lors des derniers épisodes d'écoulements torrentiels bien documentés (2012, 2015 et 2017), les dommages ont été bien inférieurs à ceux de 1987 (illustration 3), en particulier le nombre de décès qui a été considérablement réduit ${ }^{1}$.

Illustration 1 - Localisation de la zone d'étude (ville de Chosica)

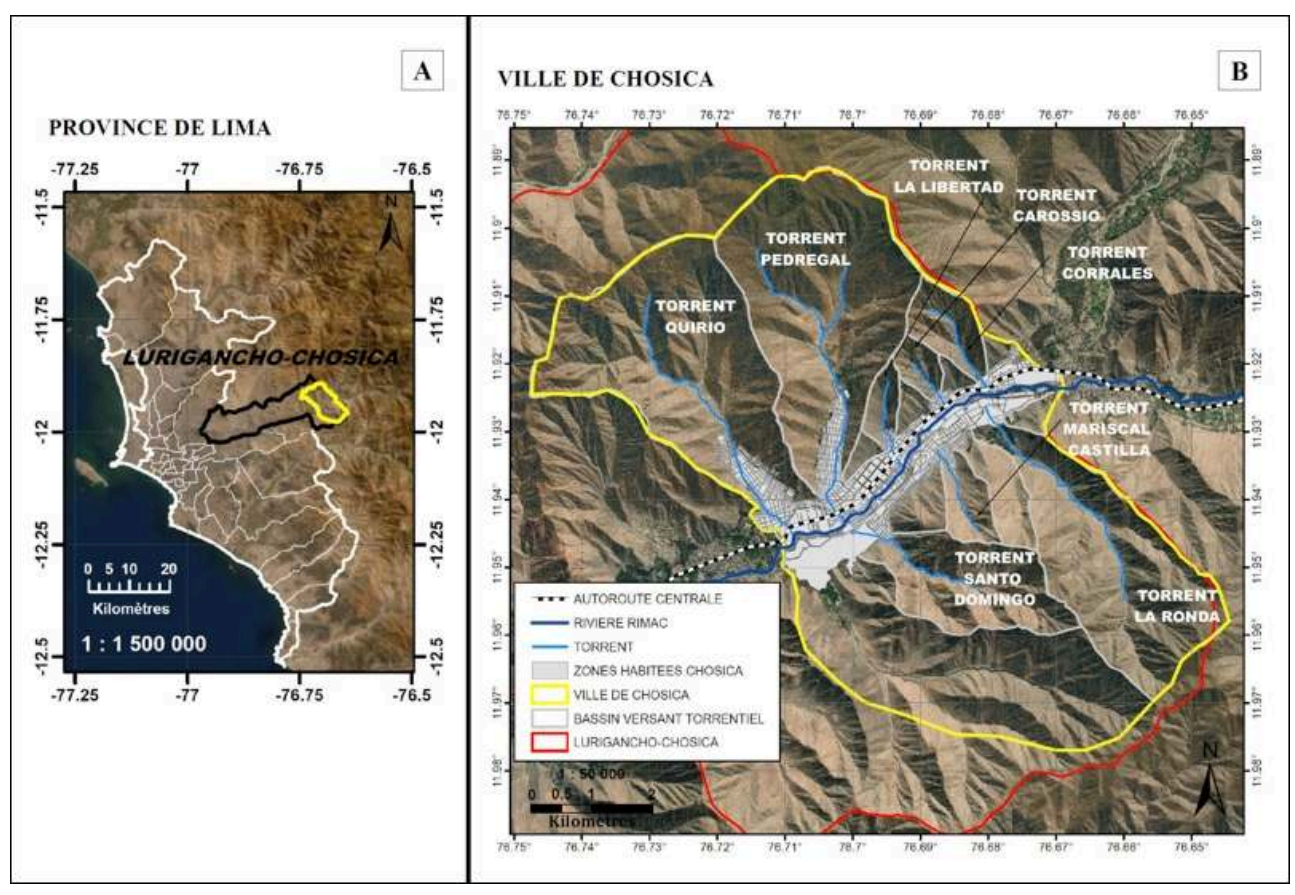

A : Localisation de la zone d'étude dans la province de Lima

B : Localisation de la zone d'étude dans le district de Lurigancho-Chosica

Sources: Google Maps (consulté le 9 mars 2018) ; DigitalGlobe, 2016 ; Mairie de Lurigancho-Chosica, 2013 ; INEI, 2007. Auteur : Alexis Mejía, mai 2018. 
Illustration 2 - Population localisée autour du ravin Pedregal sur le cône de déjection et dans la partie centrale du bassin versant torrentiel

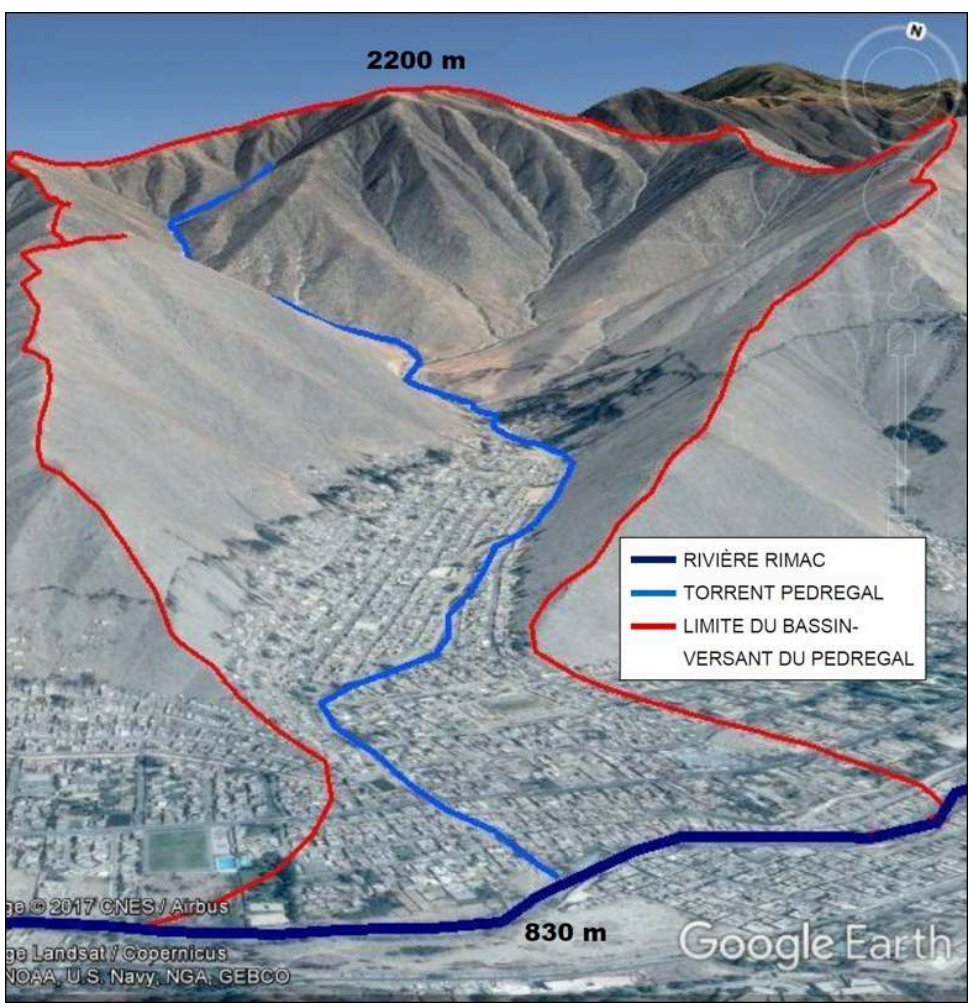

Source : Google Earth (consulté le 7 mars 2017). Auteur : Alexis Mejía, mai 2018.

Illustration 3 - Dégâts lors des écoulements torrentiels récents

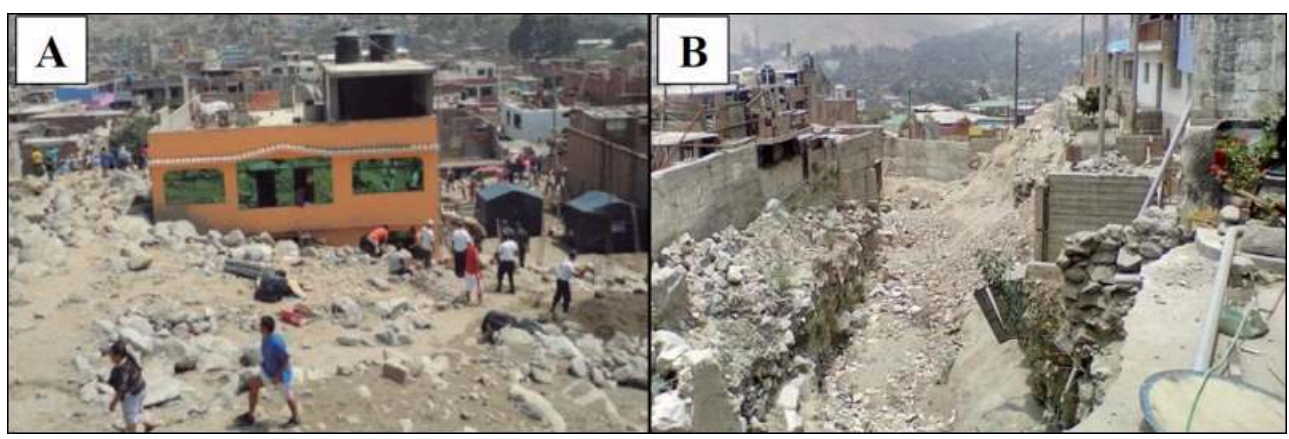

A : Dégâts dans le ravin Mariscal Castilla après les écoulements torrentiels de 2012 : seul apparait dans la photo le troisième étage d'un bâtiment enseveli du quartier Pablo Patrón.

B : Dégâts dans le ravin Quirio après les écoulements torrentiels de 2017 : logements détruits et endommagés aux côtés du lit du ravin dans le quartier Nicolás del Piérola.

Auteurs : Guadalupe et Carrillo, 2012 (A) ; Alexis Mejía, janvier 2018, 2018 (B).

4 Cet article a pour objectif d'étudier l'évolution des risques liés aux laves torrentielles dans la ville de Chosica depuis une dizaine d'années. Elle est appréhendée par l'analyse de quatre facteurs qui interviennent dans la définition du risque que retient le Centre National d'Estimation, Prévention et Réduction du Risque de Catastrophes du Pérou CENEPRED qui dépend du Ministère de la Défense : l'aléa (probabilité qu'un événement d'une certaine intensité se produise; CENEPRED, 2018), la fragilité (conditions physiques de la communauté ou de la société qui désavantagent ou affaiblissent les personnes et leurs moyens d'existence face à l'aléa; CENEPRED, 2018), le degré 
d'exposition (quantité de personnes ou de biens localisés dans un espace susceptible d'être affecté par l'aléa ; CENAPRED, 2001), et la résilience (capacité des personnes ou des systèmes à faire face au phénomène et réduire ses effets; $c f$. site FAO, visité en 2017). Nous retenons la relation suivante entre ces facteurs et le risque, conformément à celle du CENEPRED, la résilience apparaissant en dénominateur :

$$
\text { RISQUE }=\frac{A L E A * \text { FRAGILITE } * \text { DEGRE D'EXPOSITION }}{\text { NIVEAU DE GESTION DU RISQUE DE CATASTROPHES }}
$$

5 Cette définition s'approche de celle de Pigeon (2003) qui détermine le risque comme le croisement d'un aléa et d'une vulnérabilité. Cette dernière est représentée ici par la fragilité et le degré d'exposition aux aléas figurés par le nombre de personnes en zones de forts écoulements torrentiels. Cette définition a évolué, en lien avec celle de la vulnérabilité, puisque selon Metzger et d'Ercole (2011), le risque se décompose entre un enjeu (ce qui peut être perdu) et la vulnérabilité, qui inclue l'exposition aux aléas. Néanmoins, la définition adaptée du CENEPRED a été conservée.

6 La problématique de l'article est la suivante : Y a-t-il eu des variations dans les facteurs de risque (aléa, fragilité, degré d'exposition et niveau de gestion du risque) durant les trois derniers épisodes d'écoulements torrentiels dans la ville de Chosica (2012, 2015 et 2017) ? Cet article vise à actualiser et synthétiser les rares recherches sur les écoulements torrentiels à Chosica (Nuñez et al., 2015; Guadalupe et Carrillo, 2012 ; Abad, 2009) et à analyser de manière multidisciplinaire la complexité de ces événements. Il présente trois sections qui comporteront chacune une partie relative aux données et méthodes utilisées. La première section analyse l'aléa torrentiel lors des trois épisodes de 2012, 2015 et 2017 en considérant principalement le rôle du forçage pluviométrique sur le déclenchement des événements. La seconde section étudie l'évolution de la fragilité des logements et du nombre de personnes exposées à l'aléa torrentiel en utilisant les recensements généraux de la population de 1993 et 2007. La troisième section décrit des mesures de gestion du risque et cherche à quantifier l'impact de barrières flexibles placées en travers des ravins pour prévenir l'écoulement des laves torrentielles.

\section{Analyse de l'aléa torrentiel : description des écoulements torrentiels}

7 Les facteurs qui favorisent les écoulements torrentiels sont les caractéristiques physiques propres d'un bassin versant: charge sédimentaire disponible pour le transport (liée aux conditions géomorphologiques du terrain), forte pente des versants et du profil en long et précipitations (Schumm, 1973). Ci-dessous sont traitées les caractéristiques des ravins et des pluies à Chosica ainsi que la fréquence et l'intensité des écoulements torrentiels de 2012, 2015 et 2017 dans les différents ravins de Chosica et leur relation avec les précipitations quotidiennes.

8 Il est important de préciser que pour réaliser ce travail nous n'avons pas disposé de données sur les quantités de débris disponibles dans les bassins-versants et que nous n'avons pu effectuer ces mesures. Ce travail considère donc que le stock de débris n'est pas limitant et n'empêche pas la formation de laves torrentielles. Par contre la 
pluviométrie diffère d'un événement à un autre. Leur variabilité est donc prise en compte pour analyser les différences entre événements.

\section{Géomorphologie des ravins}

9 Guadalupe et Carrillo (2012) et Nuñez et al. (2015), ont bien décrit la géomorphologie des quatre ravins qui présentent le plus souvent des écoulements et produisent le plus de dommages (Quirio, Pedregal, Carossio et Corrales). Ils indiquent que ces bassins versants torrentiels sont hautement susceptibles de produire des flux de sédiments et de boue, tout comme les quatre autres ravins de la zone d'étude (illustration 1) qui ont des caractéristiques lithologiques, des pentes et des modes d'occupation du sol similaires. Dans tous les cas, la roche mère locale est constituée de roches intrusives qui sont fortement fracturées et météorisées, donc susceptibles de fournir beaucoup des débris, et les ravins ont des affluents dont les pentes sont très fortes (entre $20^{\circ}$ et $45^{\circ}$ ). Ces derniers facilitent le déplacement vers l'aval de blocs de grandes dimensions, de galets, de graviers, de sables et de limons. De plus, dans la partie inférieure et centrale des bassins versants torrentiels, les ravins sont canalisés par des murs qui ont réduit la largeur du lit pour faciliter l'urbanisation. La réduction du volume du lit augmente la vitesse des flux torrentiels, accélère l'arrivée de sédiments à l'aval ce qui accroit l'énergie des écoulements, augmentant les risques de dommages aux logements qui se situent autour des ravins. L'illustration 4A montre la géomorphologie du ravin Pedregal et de ses affluents représentatifs des ravins de la région et l'illustration $4 \mathrm{~B}$ la grande quantité de matériel instable et mobilisable dans le lit de ce torrent.

Illustration 4 - Description géomorphologique du ravin Pedregal

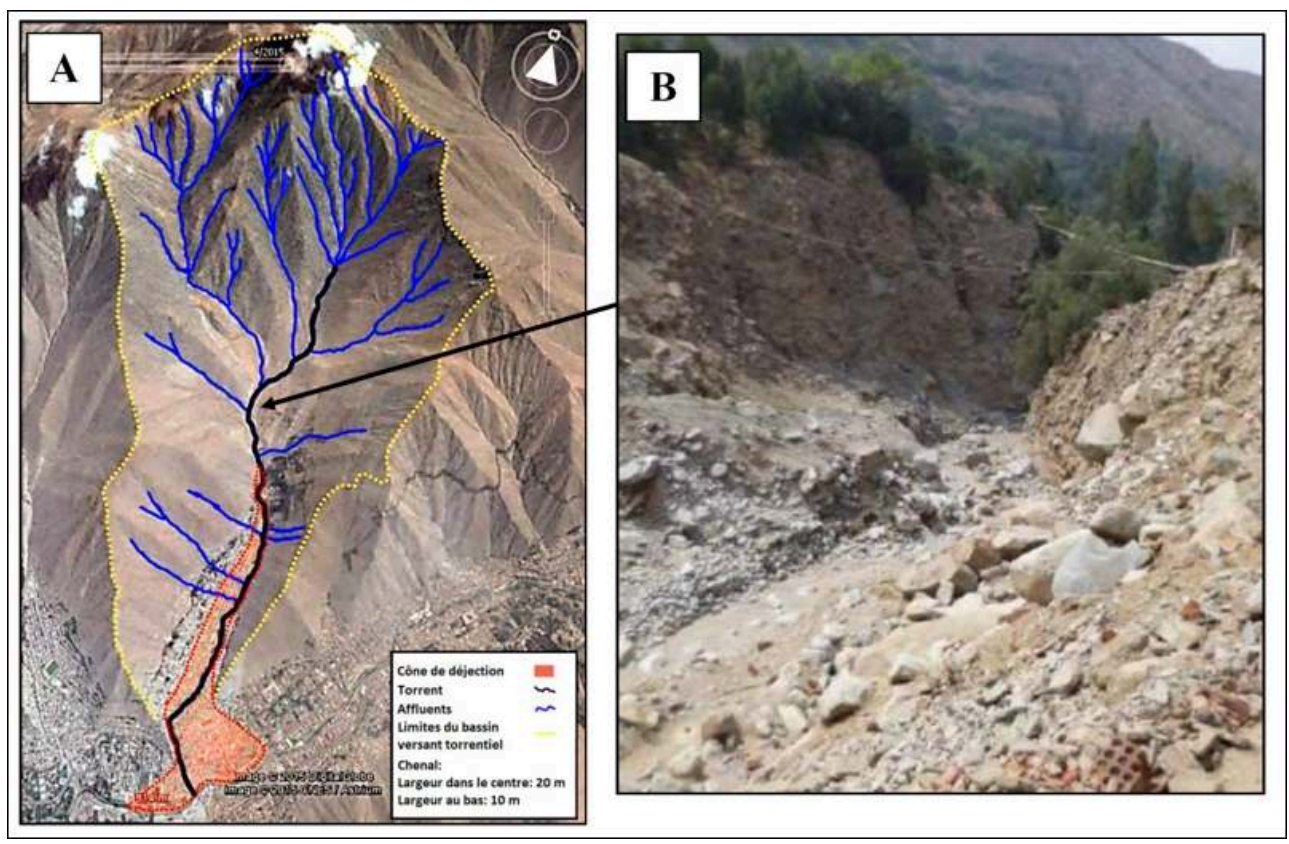

A : Bassin-versant du torrent Pedregal, ses affluents et le cône de déjection.

B : Matériel instable et mobilisable dans le lit du torrent Pedregal.

Sources : Nuñez et al., 2015 ; Auteurs : Alexis Mejía, novembre 2019 (A) ; Nuñez et al., 2015 (B) 


\section{Les pluies dans le bassin du Rimac}

10 Les précipitations à Chosica sont très faibles comme dans une grande partie de la frange côtière désertique du Pérou : $32 \mathrm{~mm}$ par an en moyenne entre 1981 et 2016 (SENAMHI, 2018b). L'aridité est principalement liée au couplage océan-atmosphère sur les côtes Pacifique tropicales d'Amérique du Sud. En effet, le courant de Humboldt, ainsi que la remontée d'eaux froides de profondeur (upwelling) renforcent l'anticyclone subtropical du Pacifique sud, ce qui stabilise l'atmosphère et empêche les précipitations sur la région côtière (Tumialán, 2015). En amont, dans les Andes tropicales, les pluies sont plus abondantes. Le total moyen annuel des précipitations dans le bassin-versant du Rimac est de $321 \mathrm{~mm}$ (1981-2016) et le débit moyen du fleuve est de $31 \mathrm{~m}^{3} / \mathrm{s}$ (1969-2004, Lavado et al., 2012).

11 Une saison plus pluvieuse a lieu en été austral, en relation avec l'arrivée d'eaux chaudes qui proviennent du Pacifique équatorial (ANA, 2010). C'est aussi le moment où il pleut le plus dans les Andes tropicales, en particulier en amont dans le haut bassin du Rímac. Même si les pluies d'été austral restent très modestes en moyenne, des pluies quotidiennes importantes permettent l'activation des torrents de Chosica, d'autant plus, comme nous l'avons dit précédemment, que la charge sédimentaire disponible pour le transport est considérable.

\section{Fréquence et intensité des écoulements torrentiels de 2012, 2015 et} 2017

\section{Classification des écoulements torrentiels}

12 La classification de Meunier (1991), qui est basée sur le type de matériau (cohésif ou granulaire) et la teneur en eau, distingue deux types d'écoulements qui sont observés à Chosica: des écoulements hyper-concentrés et des laves torrentielles qui ont des conséquences différentes en termes de dommages. La prise en compte de ces caractéristiques relatives à la composition du matériel sédimentaire d'une part, et du volume des flux torrentiels qui arrivent à l'aval d'autre part, permet d'établir quatre niveaux d'intensité du phénomène : faible, moyen, fort et très fort (tableau 1).

Les témoignages des habitants et du chef du Centre d'Opérations de Secours de Chosica (COE-CHOSICA) sur la composition du flux et les informations apportées par des documents publiés par des institutions ${ }^{2}$ ont permis d'attribuer un niveau d'intensité à tous les écoulements enregistrés en 2012, 2015 et 2017.

14 Un niveau d'intensité, faible $(=1)$, moyen $(=2)$, fort $(=3)$ ou très fort $(=4)$, a été attribué à chacun des 60 écoulements torrentiels quotidiens enregistrés entre 2012 et 2017 dans les différents ravins de Chosica. Ainsi, pour chaque date, on obtient une valeur dite d'«intensité globale» qui est la somme des intensités des événements du jour (tableau 2). Les sommes des valeurs d'intensité sont également calculées pour chaque ravin pour l'ensemble de la période d'étude. 
Tableau 1 - Niveaux d'intensité des écoulements des torrents de la ville de Chosica

\begin{tabular}{|c|c|}
\hline NIVEAUX·D'INTENSITÉø & TYPE·D'ÉCOULEMENT·TORRENTIELপ \\
\hline FAIBLEপ̆ & 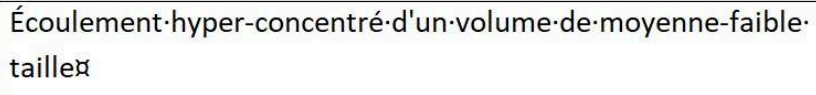 \\
\hline MODERÉপ̆ & Écoulement·hyper-concentré·d'un·gros·volumeæx \\
\hline FORTQ & 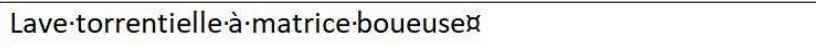 \\
\hline TRÈSFORTด् & 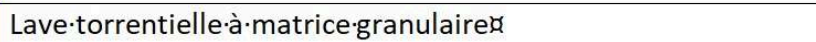 \\
\hline
\end{tabular}

Source : Meunier, 1991.

\section{Les différents événements de laves torrentielles}

Au total, 60 écoulements ont été enregistrés dans les huit ravins de Chosica, en 19 jours, entre 2012 et 2017 ( 14 jours en 2017, 4 jours en 2015 et 1 jour en 2012) (tableau 2); 48 écoulements se sont produits dans les ravins qui se trouvent sur la rive droite de la rivière Rímac, contre 12 sur la rive gauche. La différence du nombre d'écoulements entre rive droite et rive gauche ne relève pas d'une différence géomorphologique entre ces deux rives et n'est pas comprise en l'état actuel des travaux. Ce sont les ravins Pedregal, Quirio et Carossio, en rive droite, qui ont connu le plus grand nombre d'événements (13,12 et 9 respectivement). Sur 60 événements, 33 (soit $55 \%$ ) ont eu un niveau d'intensité modéré, suivi de loin par 10 qui ont eu un niveau d'intensité fort, 9 avec une intensité faible et 8 avec une intensité très forte. Pedregal, Quirio et Carossio ont eu le plus grand nombre d'écoulements d'intensité forte (3,3 et 0 , respectivement) et très forte $(1,1$ et 2 , respectivement).

Le tableau 3 nous donne une vision résumée des écoulements torrentiels dans la zone d'étude pour chaque année. Sur les 60 écoulements, quatre se sont produits en 2017 (soit 68,3\%), douze en 2015 et sept en 2012. Donc, la quantité d'écoulements dénombrés a été plus élevée en 2017, peut-être parce que leur enregistrement a été plus détaillé, incluant des écoulements très faibles, ce qui n'a peut-être pas été fait en 2012 et 2015. Cependant, l'ensemble des écoulements forts et très forts (laves torrentielles) ont été moins nombreux en 2017 (19,5\%) qu'en 2015 (50\%) et en 2012 (57,1\%). De plus, les événements des 15 et 16 mars 2017 ont été forts mais pas autant que ceux du 23 mars 2015 ou du 5 avril 2012.

17 L'indicateur d'intensité globale (somme des valeurs de l'intensité de chaque déclenchement de laves torrentielles) montre que la date à laquelle l'intensité globale a été la plus élevée est le 05/04/2012 avec une valeur de 22 (4 événements d'intensité très forte et 3 d'intensité modérée), suivie de loin par le 23/03/2015 avec une valeur de 18 ( 2 événements très forts, 2 forts et 2 modérés) et par le 25/01/2017 qui a aussi une valeur de 18 (2 événements forts et 6 modérés) (tableau 2). 
Tableau 2 - Date et intensité des écoulements torrentiels dans la ville de Chosica en 2012, 2015 et 2017

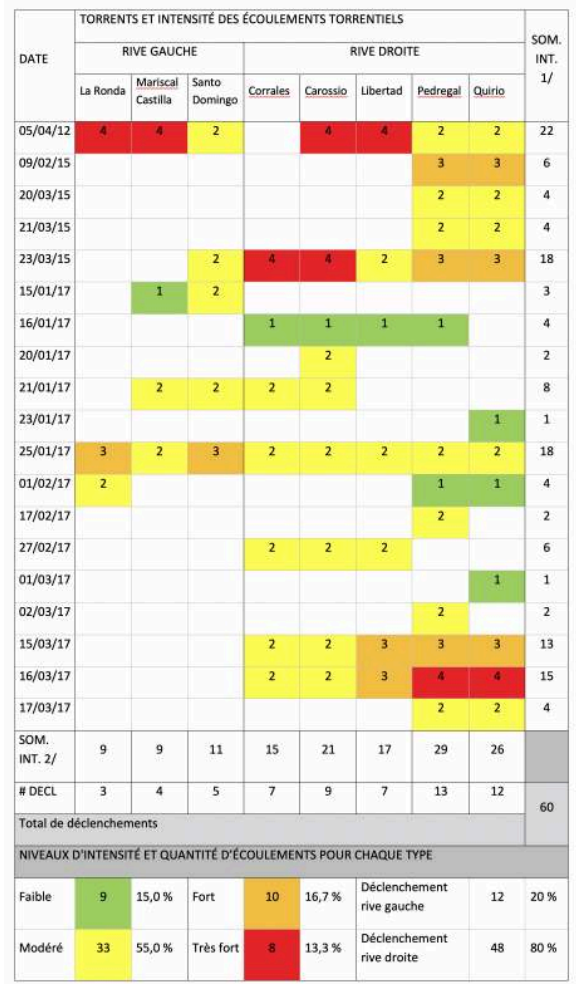

1/ Somme des intensités quotidiennes.

2/ Somme des intensités par ravin.

Sources : SINPAD-INDECI (consulté le 22 mars 2018) ; COE-CHOSICA, 2017 ; PREDES, 2015 ; Nuñez et al., 2015; Guadalupe et Carrillo, 2012.

Tableau 3 - Ensemble des écoulements torrentiels selon le niveau d'intensité dans la ville de Chosica en 2012, 2015 et 2017

\begin{tabular}{|l|l|l|l|l|l|l|}
\hline \multirow{2}{*}{ NIVEAUX D'INTENSITÉ } & \multicolumn{2}{l}{2012} & \multicolumn{2}{l|}{2015} & \multicolumn{2}{l|}{2017} \\
\cline { 2 - 8 } & Nbre & $\%$ & Nbre & $\%$ & Nbre & $\%$ \\
\hline FAIBLE & 0 & 0,0 & 0 & 0,0 & 9 & 22,0 \\
\hline MODÉRÉ & 3 & 42,9 & 6 & 50,0 & 24 & 58,5 \\
\hline FORT & 0 & 0,0 & 4 & 33,3 & 6 & 14,6 \\
\hline TRÈS FORT & 4 & 57,1 & 2 & 16,7 & 2 & 4,9 \\
\hline TOTAL & 7 & $\mathbf{1 0 0}$ & $\mathbf{1 2}$ & $\mathbf{1 0 0}$ & $\mathbf{4 1}$ & $\mathbf{1 0 0}$ \\
\hline \% (FORT + TRÈS FORT) & $\mathbf{5 7 , 1}$ & $\mathbf{5 0 , 0}$ & $\mathbf{1 9 , 5}$ \\
\hline
\end{tabular}

Sources SINPAD-INDECI (consulté le 22 mars 2018); COE-CHOSICA, 2017 ; PREDES, 2015 ; Nuñez et al., 2015 ; Guadalupe et Carrillo, 2012. 


\section{Relation entre quantité de précipitation et intensité globale des écoulements torrentiels par jour}

L'intensité globale journalière (pour les 19 jours où se sont produites les laves torrentielles) peut être liée à la quantité de pluie quotidienne enregistrée par la station de Chosica du Service National de Météorologie et Hydrologie (SENAMHI). Le manque de données infra-quotidienne ne nous a pas permis de tenir compte de l'intensité autre que diurne des précipitations. L'illustration 5 présente les valeurs journalières des pluies et de l'intensité des écoulements entre janvier et avril (JFMA) pour les années 2012, 2015 et 2017. Même s'il y a eu des pluies relativement fortes qui n'ont pas produit d'écoulement torrentiel (par exemple les 9,6 mm du 19/02/12), des pluies sont le plus souvent associées à des écoulements. Généralement des laves torrentielles de forte intensité sont observées lors de fortes pluies (plus de $10 \mathrm{~mm}$ en une journée) et elles sont moindres lorsque les pluies sont plus faibles (moins de $10 \mathrm{~mm}$ ). Une corrélation significative $(p<0,05)$ est observée entre ces deux variables pour les 19 jours qui ont présenté des écoulements $\left(R^{2}=0,791\right)$.

19 La relation entre intensité des écoulements torrentiels et précipitations montre que l'intensité de l'aléa dépend donc fortement de la quantité de pluie dans notre zone d'étude. Les quantités de pluie totales à Chosica des quatre mois de janvier à avril pour 2012, 2015 et $2017\left(62,6 \mathrm{~mm}, 61,2 \mathrm{~mm}\right.$ et $107,3 \mathrm{~mm}$, respectivement $\left.{ }^{3}\right)$ permettent de conclure que l'intensité de l'aléa pluviométrique de 2017 a été plus élevée que ceux de 2015 et 2012. On aurait donc pu s'attendre à des laves torrentielles plus fortes en 2017, contrairement à ce qui s'est produit. Pour éclairer cette contradiction apparente, il faut analyser un autre facteur qui intervient dans la variation de l'intensité de l'aléa; il s'agit des mesures structurelles destinées à réduire l'impact du phénomène naturel, sujet qui sera traité plus bas. 
Illustration 5 - Quantité de précipitation et intensité globale des écoulements torrentiels par jour entre janvier et avril pour les années 2012, 2015 et 2017

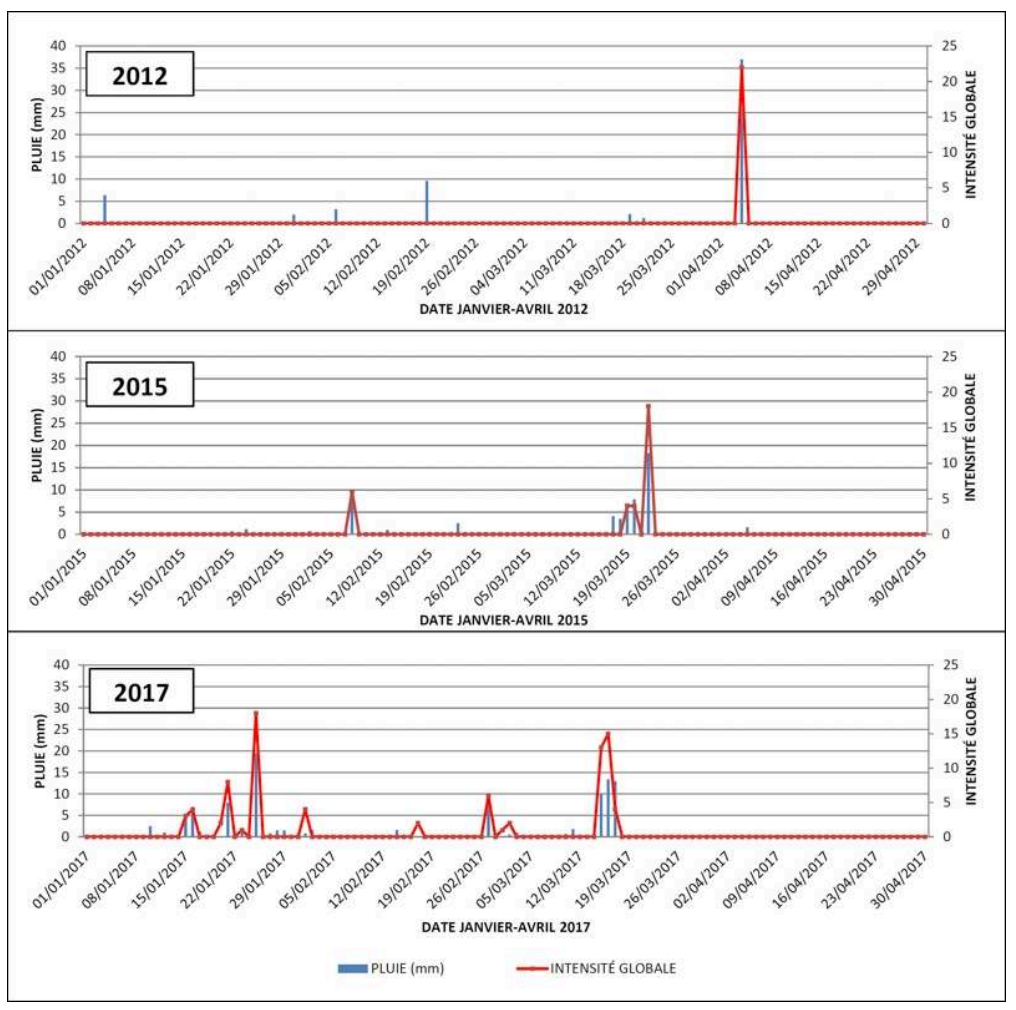

Sources : SENAMHI, 2018a ; SINPAD-INDECI (consulté le 22 mars 2018) ; COE-CHOSICA, 2017 PREDES, 2015 ; Nuñez et al., 2015 ; Guadalupe et Carrillo, 2012. Auteur : Alexis Mejía, juin 2018.

\section{La fragilité et le degré d'exposition}

La fragilité et le degré d'exposition, éléments de la vulnérabilité comprise comme la susceptibilité de la population, d'une structure physique ou d'activités socioéconomiques à subir des dommages à cause d'un aléa (CENEPRED, 2018), sont ici analysés en fonction du type d'habitat et du nombre de personnes présentes dans les ravins où les écoulements sont les plus fréquents (Perrin et al., 2000). Ils sont analysés dans une zone située sur la rive droite de la rivière Rímac au niveau de la ville de Chosica, choisie car elle comprend les 5 ravins qui ont connu les écoulements torrentiels les plus nombreux et les plus intenses entre 2012 et 2017 (tableau 2). D'autre part l'Autoroute Centrale qui se localise avant la confluence de ces ravins avec la rivière Rímac est bloquée lors de ces événements, ce qui produit de fortes pertes économiques au niveau national.

\section{Données et méthodes pour évaluer la fragilité des logements et leur degré d'exposition}

La zone retenue comprend 687 pâtés de maison selon la base de données cartographique du dernier recensement de l'Institut National de Statistique et d'Informatique du Pérou (INEI) réalisé en $2007^{4}$. Cette base de données est fiable et proche de la réalité puisque le recensement a concerné tous les logements, qu'ils soient 
formels ou non. Puisque cette analyse est appliquée aux logements, seuls les pâtés de maison à usage résidentiel ont été pris en compte. Le « Règlement d'Aménagement du Territoire et de Développement Urbain du Pérou » donne à une zone la catégorie de Zona Residencial de Densidad Baja-RDB (Zone Résidentielle de Faible Densité, en espagnol) si sa densité est de 110 hab./ha à 330 hab./ha. En effet, les densités inférieures correspondent à d'autres usages comme les commerces ou les espaces verts (DNU, 2011). Donc, l'analyse est réalisée sur 504 pâtés de maison avec une densité de population supérieure à 110 hab./ha et qui contiennent des logements.

Le matériau de construction des murs des logements est considéré par l'Institut National de la Sécurité Civile (INDECI, 2005) comme un indicateur de fragilité face à un aléa torrentiel; en fonction de ce facteur, les logements sont plus ou moins plus susceptibles d'être endommagés et détruits. Ainsi, les logements les moins vulnérables face à l'aléa sismique et à l'aléa hydrologique sont ceux construits en briques (INDECI, 2005). Les édifices en adobe sont retenus comme moins résistants. Même s'il ne s'agit pas du même type d'aléa, D'Ercole et al. (2007) indique que lors du fort séisme de 2007 dans la région de Pisco, au sud de Lima, beaucoup d'édifices en adobe se sont effondrés. On peut donc en déduire que les logements fragiles seraient plutôt ceux qui ne sont pas construits en brique.

Un indicateur de niveau de fragilité a été construit afin d'estimer le pourcentage de logement vulnérables (logement avec des murs en matériaux fragiles) par pâtés de maison. Comme le nombre de logements vulnérables peut être exprimé par la différence entre le nombre total de logements et le nombre de logements en brique, l'indicateur (pourcentage de logements vulnérables) est le suivant :

$\%$ Logements vulnérables $=\left[\frac{(\# \text { total de logements }-\# \text { logements en brique })}{\# \text { totalde logements }}\right] \times 100 \%$

La méthode de Jenks a été utilisée pour discrétiser les 504 valeurs de cet indicateur et créer quatre classes de fragilité (faible, modérée, forte et très forte). Les niveaux de fragilité des logements de la zone d'analyse sont représentés dans l'illustration 6 et le tableau 4.

L'évolution du degré d'exposition est examinée en comparant les populations de 1993 et de 2007 (dates des deux derniers recensements) localisées dans les «secteurs critiques » face à l'aléa hydrologique à Chosica. Ces secteurs critiques ont été définis en 2005 par l'INDECI en considérant les pâtés de maisons pour lesquels le risque est fort (logements fragiles nombreux, population élevée et aléas fréquent et de forte intensité), dans le projet Ciudades Sostenibles (Villes Durables, en espagnol) du Programme des Nations Unies pour le Développement (PNUD) mis en place par l'Institut National de Défense Civile du Pérou - INDECI (Sierra et al., 2009).

Dans ce travail, les secteurs critiques comprennent tous les pâtés de maisons qui se localisent dans les bassins torrentiels des quatre ravins Quirio, Pedregal, Carossio et Corrales car ils ont un niveau d'aléa fort et très fort, principalement à cause de leurs pentes et du nombre de leurs affluents. Il est ainsi possible d'analyser l'évolution du degré d'exposition en comparant la population de 1993 et de 2007 dans ces zones.

Le document de l'INDECI présente la projection de la population en 2005 en fonction des données de 1993. La population des secteurs critiques de 1993 est calculée en considérant le rapport entre la population totale des zones urbaines en 1993 (54 149 personnes) et sa projection en 2005 (70 533 personnes) fournies par le même 
document. Ainsi la population de 1993 pour chaque secteur est la population estimée pour 2005 multipliée par le facteur de proportion de la population entre les zones urbaines en 1993 et en 2005 (0,77). Grâce à ce calcul, la population totale des secteurs critiques peut-être estimée à 36520 personnes en 1993.

Illustration 6 - Niveau de fragilité des logements localisés sur la rive droite de la rivière Rímac à Chosica (2007)

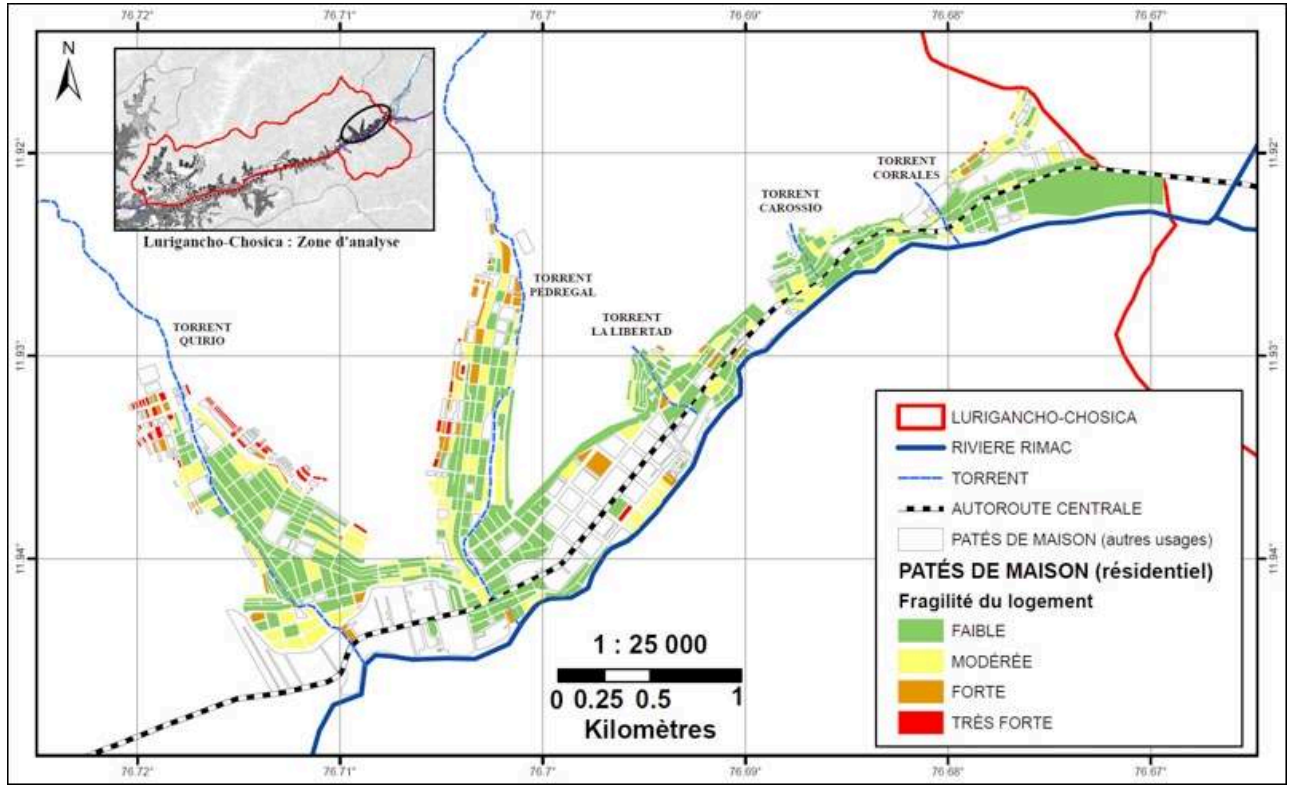

Source : INEl, 2007. Auteur : Alexis Mejía, mai 2017.

Tableau 4 - Fragilité des logements de la zone d'analyse (rive droite de la rivière Rímac à Chosica)

\begin{tabular}{|c|c|c|c|}
\hline NIVEAUXם & 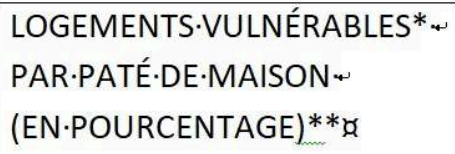 & $\begin{array}{l}\text { PATÉS-DE-MAISON } \\
\text { (RESIDENTIEL) } ₫\end{array}$ & $\begin{array}{l}\% \cdot \\
\text { TOTALX }\end{array}$ \\
\hline FAIBLEם & {$[0.0 \cdot-13.6] \not$} & $283 \not$ & $56.2 \not$ \\
\hline MODÉRÉEপ̆ & ]13.6•-37.5]ם & $117 \not x$ & $23.2 \not$ \\
\hline FORTEপ & ]37.5--.71.4]ם & $57 \not \varnothing$ & $11.3 \not a$ \\
\hline TRÈS-FORTEপ̆ & ]71.4•-100.0]ם & $47 \not x$ & $9.3 \not x$ \\
\hline 'TOTAL'요 & & $504 \not$ & $100.0 \not$ \\
\hline
\end{tabular}

* Logements avec des murs en matériaux fragiles (adobe, mur végétal en paille ou roseaux, entre autres).

** Classification par la méthode de Jenks.

Source : INEI, 2007. 


\section{Les pâtés de maison vulnérables} au niveau du district de Lurigancho-Chosica représente $72 \%$ du total (INEI, 2007). Le taux de constructions en briques a augmenté au cours du temps car selon les recensements de 1993 et 1981, leurs pourcentages étaient respectivement de 59,8 \% (INEI, 1993) et de 59,4\% (INEI, 1981). Ceci montre qu'il y a eu une réduction des logements vulnérables. En conclusion, le niveau de fragilité des logements diminue dans le temps, du moins jusqu'en 2007, et les logements fragiles se localisent dans des zones en pente.

\section{Degré d'exposition}

Les illustrations 7 et 8 montrent tous les secteurs critiques (les zones de risque fort selon l'INDECI en 2005) et la population concernée par chacun d'eux, tant pour la zone d'analyse du projet de l'INDECI (la ville de Chosica) avec la base de données de 1993 (illustration 7), que pour la zone d'analyse sur laquelle nous travaillons (rive droite de la rivière Rimac à Chosica) avec la base de données de 2007 (illustration 8).

Dans ces illustrations, il est possible d'identifier les secteurs critiques qui seraient affectés par le déclenchement de laves torrentielles dans les quatre ravins choisis (secteurs I, II, VI, VII, VIII, IX et X) aux deux dates. Pour une comparaison plus homogène, tous les pâtés de maison qui se trouvent dans ces secteurs, résidentiels ou autres, sont pris en compte pour calculer la population concernée.

Ainsi, la population affectée par le déclenchement des torrents Quirio, Pedregal, Carossio et Corrales en 1993 est estimée à 27028 personnes (habitant à la fois les pentes et les fonds de thalweg) alors que pour l'année 2007, elle était de 27947 personnes. Durant la période de 15 ans qui sépare les deux dates, le nombre de personnes affectées a donc augmenté de 919, soit une hausse de 3,3\%. Cette valeur est inférieure à celle du district $(3,8 \%)$; en effet au niveau du district l'accroissement est plus important car les espaces libres disponibles pour de nouveaux habitants sont plus nombreux qu'au niveau des ravins.

Si l'on considère que le taux d'accroissement de la population du district a été constant, de 3,8 \% entre 1981 et 1993 et entre 1993 et 2007 (INEI, 1981, 1993, 2007), on peut aussi déduire que la population affectée a augmenté dans les années 2010. A partir des 
données de 1993 et 2007, le taux d'accroissement de population dans les secteurs critiques est $0,2 \%$; si l'on applique ce taux, on trouve une population de 28691 habitants dans les secteurs critiques en 2018 (soit une augmentation de 744 personnes par rapport à 2007).

Illustration 7 - Degré d'exposition face à l'aléa hydrologique dans les secteurs critiques I à XVIII à Chosica en 1993

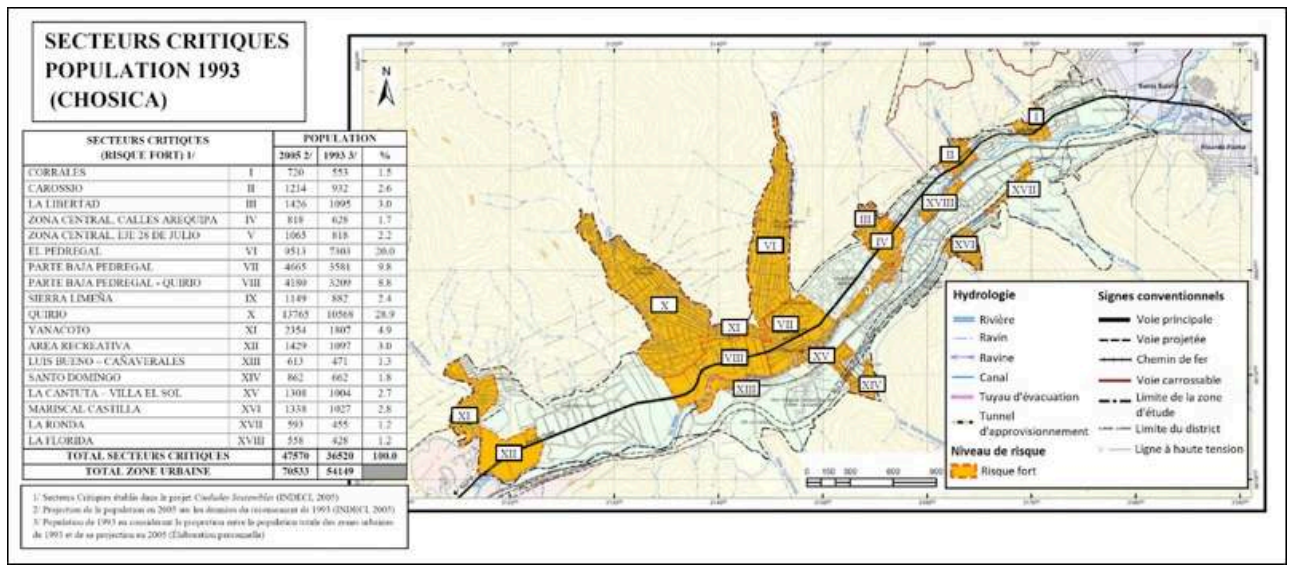

Source : INDECI, 2005. Alexis Mejía, mai 2017.

Illustration 8 - Degré d'exposition face à l'aléa hydrologique dans les secteurs critiques I, II, VI, VII, VIII, IX et X à Chosica en 2007

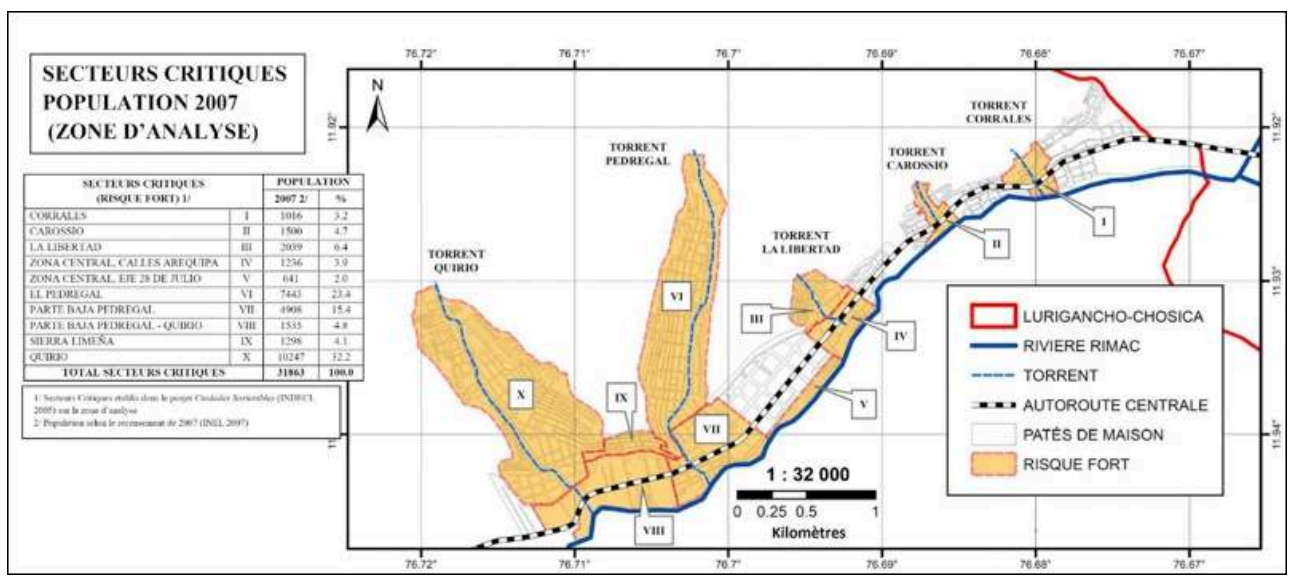

Source : INEI, 2007. Alexis Mejía, mai 2017

\section{Mesures pour la gestion du risque}

À la différence de villes comme Quito et La Paz, Lima n'a pas eu pendant longtemps de politique publique de gestion des risques qui concerne l'ensemble de l'agglomération (Sierra et al., 2009). Le Pérou s'est toutefois doté de la loi 29664 de 2011 sur la gestion des risques et d'un Plan National de Gestion des Risques de Catastrophes (PLANAGERD) pour la période 2014-2021.

Ces mesures sont de deux grands types. Les mesures structurelles ou actives sont destinées à réduire le niveau de l'aléa; elles impliquent une construction physique (ouvrages de protection comme des murs ou des digues) et elles sont liées à la 
technologie, à la connaissance et à l'accord des autorités qui les construisent. Les mesures non-structurelles ou passives ont pour objectif la prévention et la réduction de l'exposition aux risques (changement d'occupation des sols, systèmes d'alerte et d'évacuation, entre autres) (Huebl and Fiebiger 2005 cité par Rodriguez-Morata et al., 2019 ; García. 2007).

\section{Mesures non-structurelles}

.

Des mesures non-structurelles nous ont été relatées lors de visites de terrain en janvier et février 2017 et 2018, essentiellement par Monsieur José Martinez, chef du COECHOSICA. Nous avons eu également recours à différents documents administratifs ou publiés par des ONG. Nous exposerons trois mesures structurelles qui sont utilisées pour tenter de réduire le risque : la contrainte en matière d'occupation du sol pour éviter celle-ci en zones d'aléa fort, la formation des autorités et de la population à la gestion du risque, et un système d'alerte précoce (SAP) afin de prévenir la population de la possible imminence d'un événement. Cependant, hormis quelques témoignages illustratifs, nous n'avons pas d'information scientifique sur leur compréhension et leur acceptation par la population et donc sur leur pertinence et leur succès.

Même si les zones d'aléa fort ont été identifiées en 2005 dans le projet du PNUD-INDECI Ciudades Sostenibles, ce n'est que récemment, en 2012, que des actions ont été formalisées pour interdire de nouveaux établissements de population dans ces zones, entre autres pour éviter leur exposition à l'aléa torrentiel. Ainsi, les autorités de la mairie de Lurigancho-Chosica, conjointement avec des membres de la Police Nationale du Pérou (PNP), ont pu démanteler de nouvelles invasions ${ }^{5}$ dans des zones d'aléa fort $\mathrm{du}$ district (ravins California et Yanacoto) début $2017^{6}$. Cependant, l'expulsion de personnes qui vivent depuis de nombreuses années dans les zones d'aléa fort est compliquée. Les recommandations faites dans le cadre de Ciudades Sostenibles sont restées sans effet (Sierra et al., 2009) et le nombre de personnes finalement expulsées n'est pas connu, principalement à cause de l'enracinement de la population dans son territoire (PREDES, 2015). Il est probable que contrairement aux intentions initiales la population ait légèrement augmenté dans les zones d'aléa fort entre 2012 et 2017, non pas à cause de nouvelles installations, mais à cause de l'accroissement de la population déjà présente, ce qui a accru la densité de population.

La formation à la gestion du risque a été renforcée à partir de 1998 par la création d'un groupe de volontaires appelé «brigades de secours». Les membres de ce groupe se forment auprès de personnes du Corps Général des Pompiers Volontaires du Pérou (CGBVP), d'infirmiers, et de personnel qualifié de la mairie de Lurigancho-Chosica. Ces personnes seraient un appui sérieux pour faire connaître l'importance de la gestion du risque dans leurs quartiers et leur nombre augmente au fil des ans. Il est en effet possible que la population se mette plus volontiers à l'écoute de personnes voisines que des autorités ${ }^{7}$.

Depuis la catastrophe de 1987, de nombreuses initiatives ont été mises en place pour informer les populations, notamment les nouveaux arrivants, sur les risques de catastrophes et pour les préparer à réagir de façon adéquate. Par exemple, après l'épisode de 2015, le projet du Disaster Preparedness ECHO programme- DIPECHO (Programme de préparation aux catastrophes d'ECHO) a organisé différents ateliers (DIPECHO, 2016). De plus, avec l'appui de l'ONG Soluciones Prácticas (Solutions Pratiques, 
en espagnol) et de la mairie de Lurigancho-Chosica, DIPECHO a créé un événement en septembre 2016 pendant lequel les participants ont reçu une formation de manière ludique et certains, par tirage au sort, ont reçu des « sacs à dos d'urgence ", équipés des objets nécessaires pour faire face aux catastrophes (illustration 9A) (site Facebook de la Mairie de Lurigancho-Chosica, consulté le 15 mai 2017).

Finalement, un système d'alerte précoce (SAP) a été mis en place par l'ONG Soluciones Prácticas et $\mathrm{KOICA}^{9}$ en octobre 2016 pour prévenir la population de l'occurrence d'écoulements torrentiels dans quatre ravins de Chosica: Carossio, La Libertad, Corrales et Pedregal. Des pluviomètres situés dans les parties hautes de ces ravins déclenchent des avis d'alerte à la population des ravins et au COE-CHOSICA quand ils enregistrent des fortes quantités de pluies (illustration 9B). Deux niveaux d'alerte ont été définis : une sirène émet par intermittence quand la quantité de pluie d'une journée dépasse $3 \mathrm{~mm}$ et elle émet de façon continue quand la pluie dépasse $8 \mathrm{~mm}^{10}$. Cependant, le système d'alerte précoce n'est pas toujours efficace, car des alertes non suivies d'écoulement ont fait perdre à ce système un peu de sa crédibilitée ${ }^{11}$.

Illustration 9 - Mesures non structurelles pour la gestion du risque à Chosica

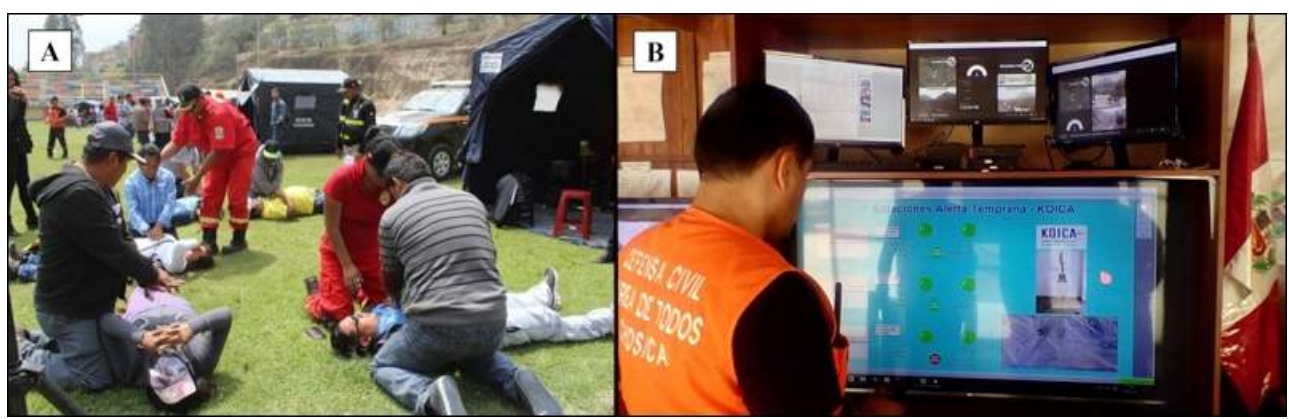

A : Événement de préparation et de formation «Brigadier d'un jour » en 2016. La population reçoit une formation, de manière pratique, pour faire face aux catastrophes. Elle est organisée par DIPECHO, I'ONG Soluciones Prácticas et la mairie de Lurigancho-Chosica.

$B$ : Système d'Alerte Précoce (SAP) à Lurigancho-Chosica. Le chef du COE-CHOSICA explique que ce système est basé sur l'installation de pluviomètres dans la partie haute de chaque bassin versant torrentiel (ravins Carossio, La Libertad, Corrales et Pedregal) et annonce les fortes pluies au bureau du COE-CHOSICA et à la population des quartiers concernées.

Auteurs : site Facebook de la Mairie de Lurigancho-Chosica, consulté le 15 mai 2017 (A) ; Alexis Mejía, 22 février 2017 (B)

\section{Mesures structurelles}

41 Depuis deux décennies environ, des digues et des murs sont construits afin de réguler les pentes, diminuer la vitesse des flux, retenir le matériel solide transporté et protéger les habitations et infrastructures du flux torrentiel. Des opérations de nettoyage des débris sont exécutées dans les ravins afin d'éviter qu'ils puissent être mobilisés lors d'un prochain événement. De plus, depuis début 2016, des barrières flexibles ont été installées en travers des ravins afin de retenir les débris entrainés par les laves torrentielles.

Les informations relatives aux mesures structurelles proviennent de visites de terrain effectuées en janvier et février 2017 et 2018 à Chosica, au cours desquelles le premier auteur a pu s'entretenir avec les autorités de la municipalité de Lurigancho-Chosica, le 
chef du COE-Chosica, le responsable des travaux de nettoyage des barrières flexibles, et des leaders de quartiers ${ }^{12}$.

De plus des documents administratifs et des articles de journaux ont été consultés. Enfin l'Autorité Nationale de l'Eau du Pérou (ANA), responsable de l'installation et du nettoyage des barrières flexibles, a fourni des données sur le volume de matériel retenu en 2017 par les 21 barrières de Chosica. La quantité de matériel retenu a été calculée compte tenu du volume du lit du ravin à une certaine distance en amont de la barrière. Ces volumes ont été classées en nul, faible, moyen, élevé et très élevé (tableau 5) selon la méthode de Jenks. Le ratio entre le volume retenu par chaque barrière et la superficie du bassin versant torrentiel $\left(\mathrm{m}^{3} / \mathrm{km}^{2}\right)$ a été mis en relation avec l'intensité des écoulements dans les ravins en 2017 (tableau 2) afin de vérifier si ces barrières ont eu l'effet escompté.

\section{Les digues et le nettoyage des ravins}

Les digues (maçonneries en pierres) et les murs (de pierres et de béton) (illustration 10) ont été construits à partir des années 1990 dans les ravins Quirio et Pedregal (Abad, 2009 ; Sierra et al., 2009) et plus tard, dans tous les ravins de la rive droite (Nuñez et al., 2015). Le long des ravins de la rive gauche de la rivière Rímac, seuls des murs de canalisation ont été construits jusqu'en 2010 environ dans quelques parties des ravins car les écoulements torrentiels n'étaient pas très fréquents dans ces zones. Cependant, en 2012, ces torrents se sont déclenchés et des dommages considérables s'y sont produits (Guadalupe et Carrillo, 2012). Après cet événement, selon les déclarations du maire de Lurigancho-Chosica, une grande partie du budget municipal a été dédié à la construction d'autres digues. En 2013, il y en avait 64 dans tout le district (Peru21, le 23 janvier 2013).

En 2015, quand se sont activés les torrents de la rive droite de la rivière Rímac, les murs et les digues ont réduit les dommages (même s'il y a eu de fortes pertes en vies humaines et économiques), mais elles ont été fortement abimées et n'étaient pas réparées lorsque s'est produit l'événement de 2017. Elles n'y ont donc pas résisté (illustration 10A) (Nuñez et al., 2015). De plus, L'événement de 2015 a occasionné la construction d'une nouvelle série de digues grâce à une convention entre la mairie de Lurigancho-Chosica et le Ministère de Logement, Construction et Assainissement du Pérou - MVCS; ces nouvelles digues ont mieux résisté à l'événement de 2017 (illustration 10B).

Les travaux de nettoyage et de décompactage des ravins sont importants pour éviter que du matériel soit disponible lors d'un événement ultérieur. Ces travaux, de la responsabilité de la mairie de Lurigancho-Chosica, sont réalisés après chaque épisode d'écoulement torrentiel. Ils ont nécessité en 2017 la mobilisation des personnels et engins de la mairie, du Ministère du Logement, Construction et Assainissement du Pérou (MVCS) et des Forces Armées (illustration 11). La population y a contribué aussi, comme elle a participé au renforcement des murs après les premiers écoulements de $2017^{13}$

Ceci montre que les autorités comme la population, si elles sont organisées, s'efforcent de nettoyer le chenal des ravins, mais elles le font essentiellement dans la partie inférieure et centrale des bassins versants torrentiels. Or, les parties hautes, bien plus difficiles d'accès, ne sont pas l'objet de nettoyage ce qui en limite l'efficacité. 
Illustration 10 - Mesures structurelles pour la gestion du risque à Chosica (digues)

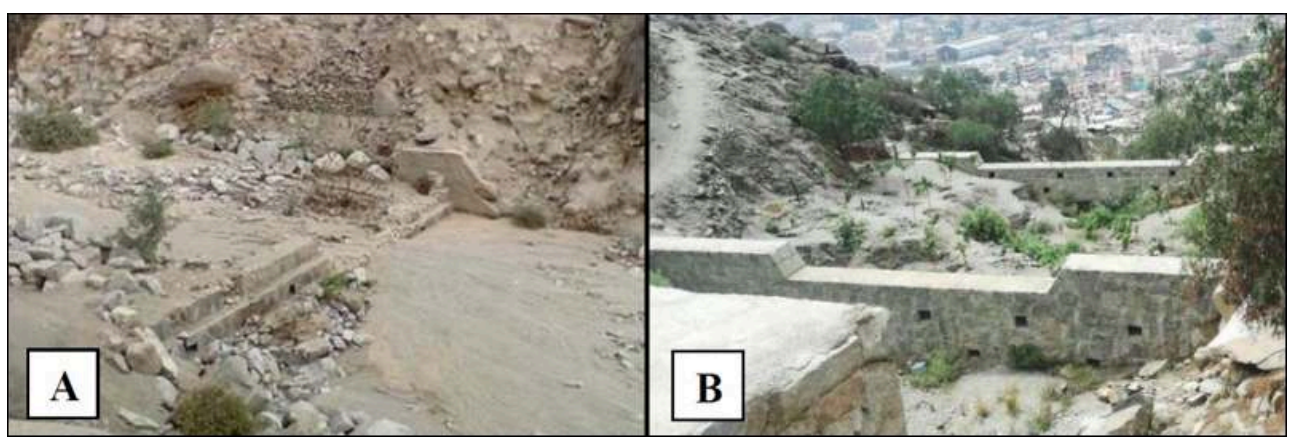

A : Digue détruite dans le ravin Pedregal.

$B$ : Digues en bon état dans le ravin Mariscal Castilla.

Auteurs : Alexis Mejía, janvier 2017 (A) ; site Facebook de la Mairie de Lurigancho-Chosica, consulté le 15 mai 2017 (B).

Illustration 11 - Mesures structurelles pour la gestion du risque à Chosica

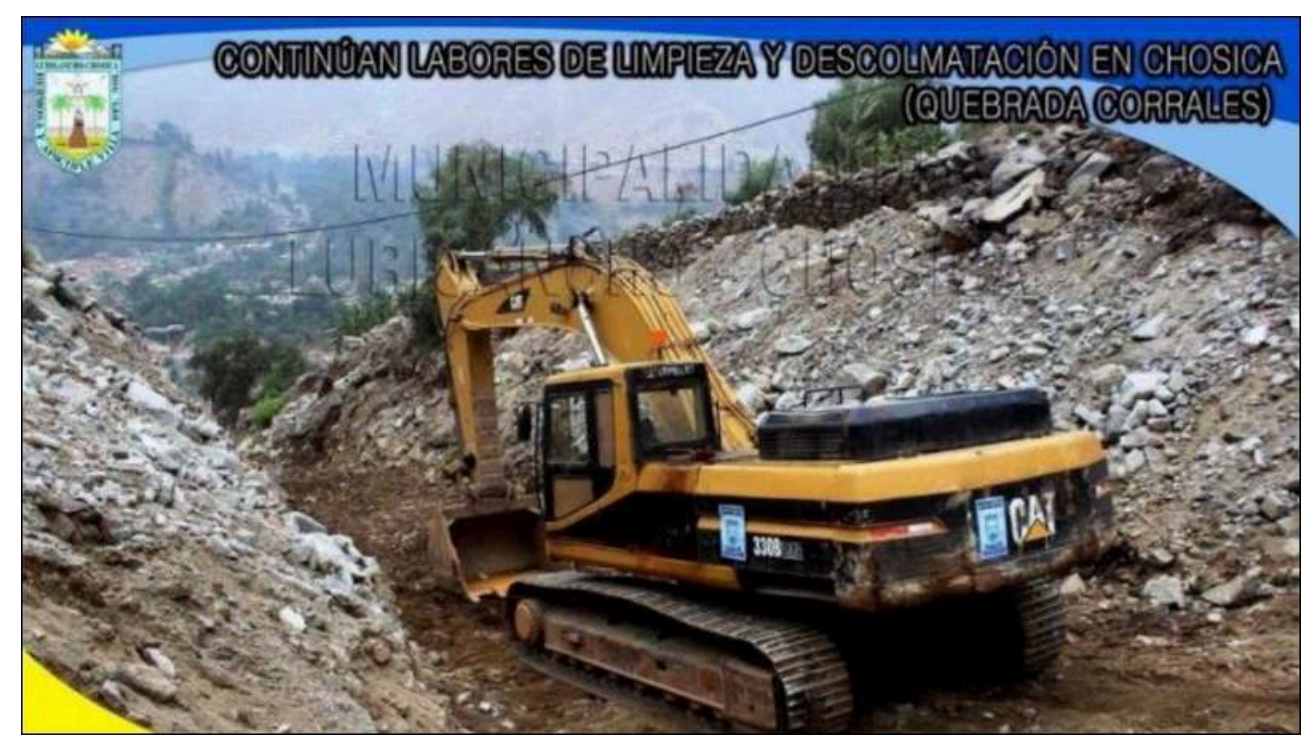

Nettoyage du ravin Corrales après les écoulements torrentiels du 25 janvier 2017.

Auteur : site Facebook de la Mairie de Lurigancho-Chosica, consulté le 15 mai 2017.

\section{Les barrières flexibles}

Une mesure novatrice au niveau national a été l'installation de Barreras Dinámicas (appelées ici, «Barrières Flexibles ${ }^{14}$ ) début 2016 par l'Autorité Nationale de l'Eau du Pérou (ANA). Ce sont des filets métalliques avec une forte capacité de déformation, installés dans la partie supérieure des bassins versants torrentiels qui ont pour fonction de retenir les matériaux transportés par les écoulements torrentiels, et d'éviter la chute de grands blocs dans les zones basses où se trouve la population (ANA, 2015a). À Lima, 22 barrières flexibles ont été installées (coût total : 21,5 millions de nuevos soles ${ }^{15}$, soit 6,5 millions de dollars; ANA, 2015b) : une dans le district voisin de chaclacayo et 21 dans les huit ravins de la zone d'étude à Chosica. Ces barrières ont des 
caractéristiques différentes en terme de dimensions et de capacité de résistance à l'impact (illustrations 12 et 13).

Illustration 12 - Barrière flexible $n^{\circ} 10$ dans le ravin Carossio

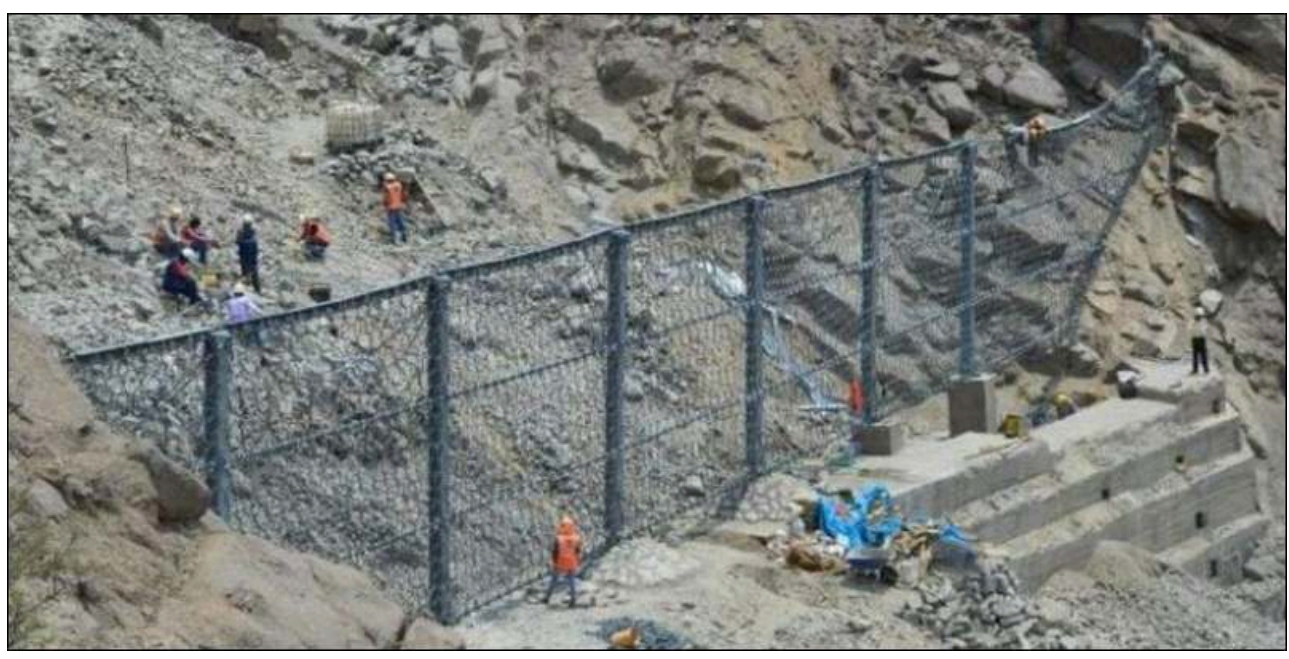

Barrière de type UX d'une longueur de $54 \mathrm{~m}$ avec six supports intermédiaires et une capacité d'impact de 180 kN/m², la plus longue des 21 barrières installées à Chosica.

Auteur: ANA, 2016.

Illustration 13 - Localisation des 21 barrières flexibles installées dans la ville de Chosica

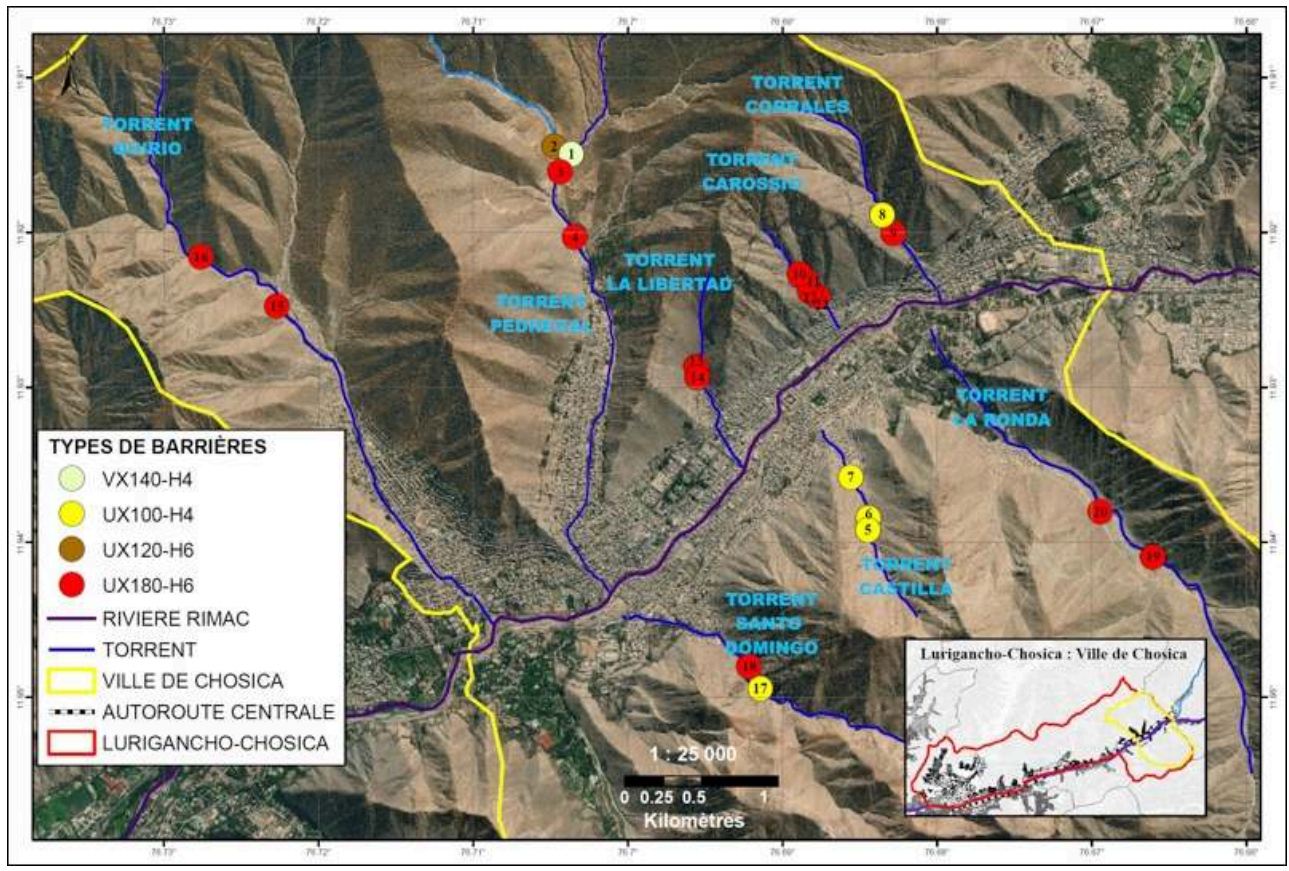

Sources : Geobrugg (consulté le 18 avril 2018) ; ANA, 2017 ; DigitalGlobe, 2016. Auteur : Alexis Mejía, juin 2018

En 2017, les barrières ont atteint leur objectif de retenir de grandes quantités du matériel issu des écoulements torrentiels (illustrations 14), qui, en leur absence, seraient arrivées à l'aval des ravins, là où se localise la population, produisant des dommages considérables. Cependant, quelques-unes ont subi des dommages surtout au 
niveau des ancrages, des éléments de protection contre l'abrasion et dans la partie inférieure des barrières (illustration 15). Il est notable cependant que les barrières flexibles n'ont pas été détruites, comme le sont souvent les digues qui sont plus rigides $^{16}$.

L'ANA, responsable des barrières flexibles, a réalisé début 2018 des travaux de nettoyage du matériel retenu par les barrières et a renforcé certains de leurs éléments.

Illustration 14 - Matériel retenu par les barrières flexibles après les écoulements torrentiels de 2017

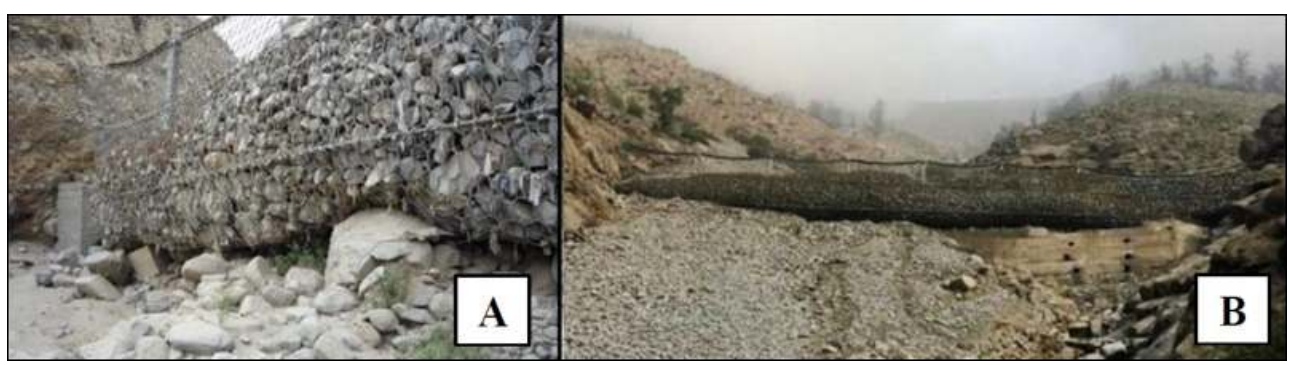

A : Barrière flexible 15 dans le ravin Quirio.

$B$ : Barrière flexible 10 dans le ravin Carossio.

Auteur : ANA, 2018b.

Illustration 15 - Dommages subis par la barrière $n^{\circ} 15$ dans le ravin Quirio

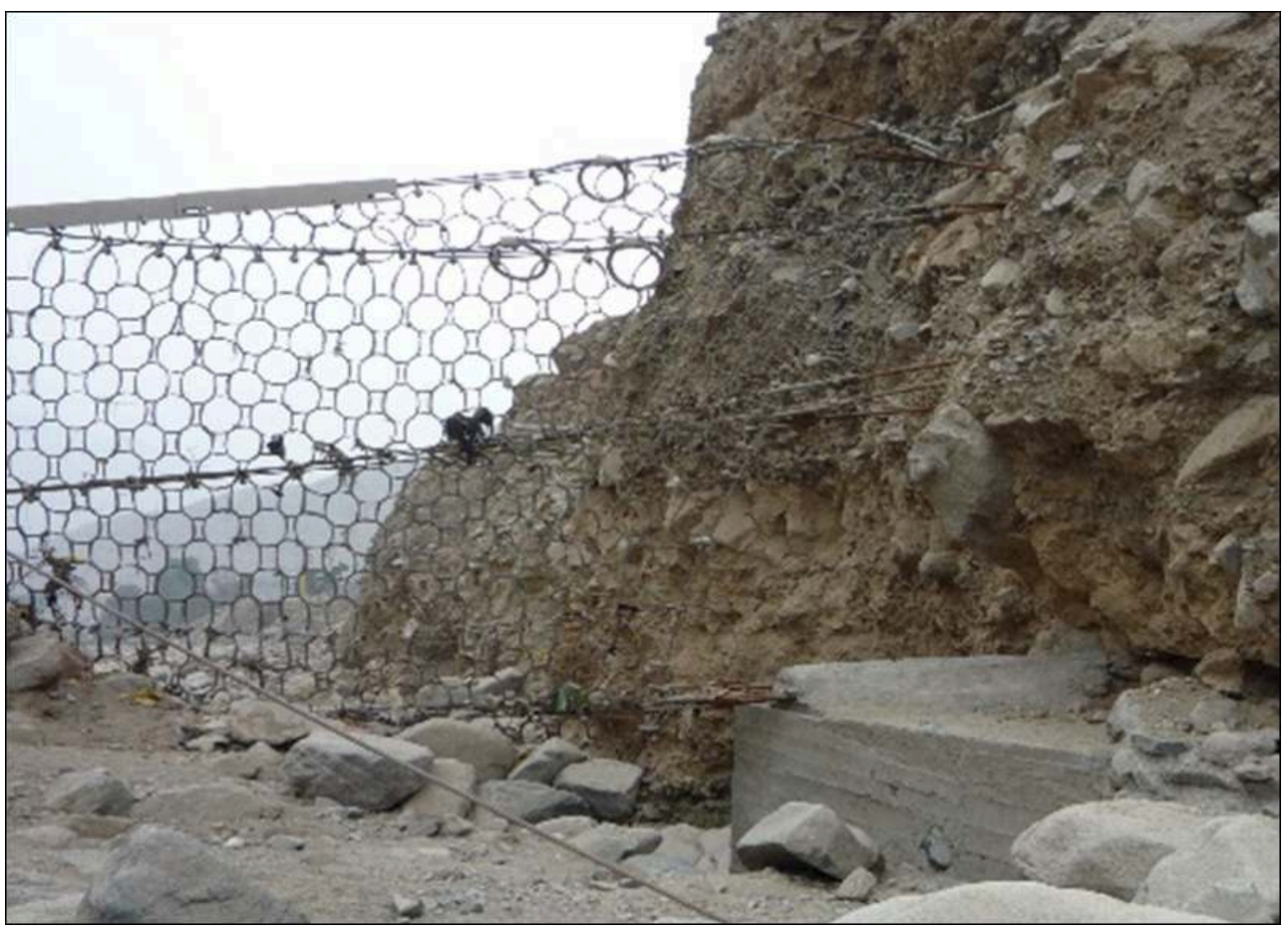

Auteur : ANA, 2018b.

Plus du tiers $(35,1 \%)$ du coût total des travaux réalisés par l'ANA en 2018 (environ 3 millions de nuevos soles, environ 900000 dollars), correspond aux travaux réalisés à Carossio, suivi de loin par le coût des travaux dans le ravin Pedregal (presque $20 \%$ du total du coût), dans le ravin La Ronda (16,6\%), et dans le ravin Quirio 15,0\%), le pourcentage étant inférieur à $4 \%$ du coût dans chacun des autres ravins (ANA, 2018a). 
52 Ces coûts ont été liés essentiellement aux travaux de nettoyage, qui dépendent principalement de deux facteurs. D'une part, ils sont dus à la très forte pente et au manque de voie carrossable qui rendent difficiles l'accès de machines et d'outils jusqu'aux lieux à dégager. D'autre part les couts élevés sont liés au volume de sédiments à déplacer ${ }^{17}$.

Le total de matériel retenu par les barrières en 2017 a été de $14837 \mathrm{~m}^{3}$ (ANA, 2017). L'illustration 16 montre que deux barrières ( 3 et 15) ont retenu les volumes les plus importants (environ $3200 \mathrm{~m}^{3}$ et $2900 \mathrm{~m}^{3}$ respectivement), et les deux suivantes (10 et 20) des volumes élevés (environ $2300 \mathrm{~m}^{3}$ et $1700 \mathrm{~m}^{3}$ ).

Des volumes moyens et faibles ont été retenus par 4 et 5 barrières, respectivement, et 8 barrières n'ont pas retenu de matériel (volume nul). Il est possible que les barrières 18 (à Santo Domingo) et 4 (à Pedregal) n'aient pas retenu de matériel car les barrières situées en amont l'ont fait. Pour les cinq autres barrières, situées elles en aval, il est possible que le matériel se soit formé entre ces barrières et la plus proche en amont, issu des affluents du ravin, ou de l'érosion du lit du ravin.

Les ravins Mariscal Castilla, La Libertad et Santo Domingo et Corrales, n'ont pas transporté de matériel sédimentaire (les volumes retenus au niveau du ravin n'atteignant pas $1 \%$ du matériel retenu total). Au contraire, les ravins qui ont retenu la plus grande quantité ont été Pedregal $\left(29,6 \%\right.$ du total, $\left.14837 \mathrm{~m}^{3}\right)$, suivi de Quirio (27,0 \%), Carossio (21,8 \%) et La Ronda (20,7 \%).

Le ratio entre le volume retenu et la superficie du bassin versant torrentiel de ces derniers ravins est de $439 \mathrm{~m}^{3} / \mathrm{km}^{2}$ à Pedregal, 363 à Quirio, 6464 à Carossio et 409 à La Ronda. La valeur de Carossio est très supérieure aux autres, car sa surface est presque vingt fois plus petite que celles des autres et sa pente est très forte, atteignant $40 \%$. Cette conclusion est formulée sous réserve que la quantité de débris disponibles pour le transport soit la même dans les quatre bassins versants torrentiels. Cette estimation est assez grossière ; cependant, il est clair que la pente joue un rôle important dans cette analyse.

Tableau 5 - Classification des barrières flexibles à Chosica selon le volume retenu lors des écoulements torrentiels de 2017

\begin{tabular}{|c|c|c|c|}
\hline VOLUME囚 & CLASSES* $\cdot\left(\mathrm{m}^{3}\right) \not$ & $\begin{array}{c}\text { NBRE·DE. } \\
\text { BARRIÈRESQ }\end{array}$ & $\%$ TOTALX \\
\hline NULX & $0 \not x$ & $8 x$ & 38,10 \\
\hline FAIBLEם & {$[10,0 \cdot-\cdot 100,0] \not$} & $5 \not$ & $23,8 \propto$ \\
\hline MOYENם & {$[100,1 \cdot \cdots \cdot 1500,0] \not$} & $4 \not x$ & $19,0 \not x$ \\
\hline ÉLEVÉવ̆ & {$[1500,1 \cdot-2500,0] \varnothing$} & $2 x$ & $9,5 \not$ \\
\hline TRÈS-ÉLEVÉq & {$[2500,1 \cdot-3212,4] \not ్$} & $2 \not x$ & 9,5 व \\
\hline \multicolumn{2}{|c|}{ TOTALX } & $21 \alpha$ & $100,0 x$ \\
\hline
\end{tabular}

* Classification par la méthode de Jenks.

Source : ANA, 2017. 
Illustration 16 - Barrières flexibles à Chosica selon le volume retenu lors des écoulements torrentiels de 2017

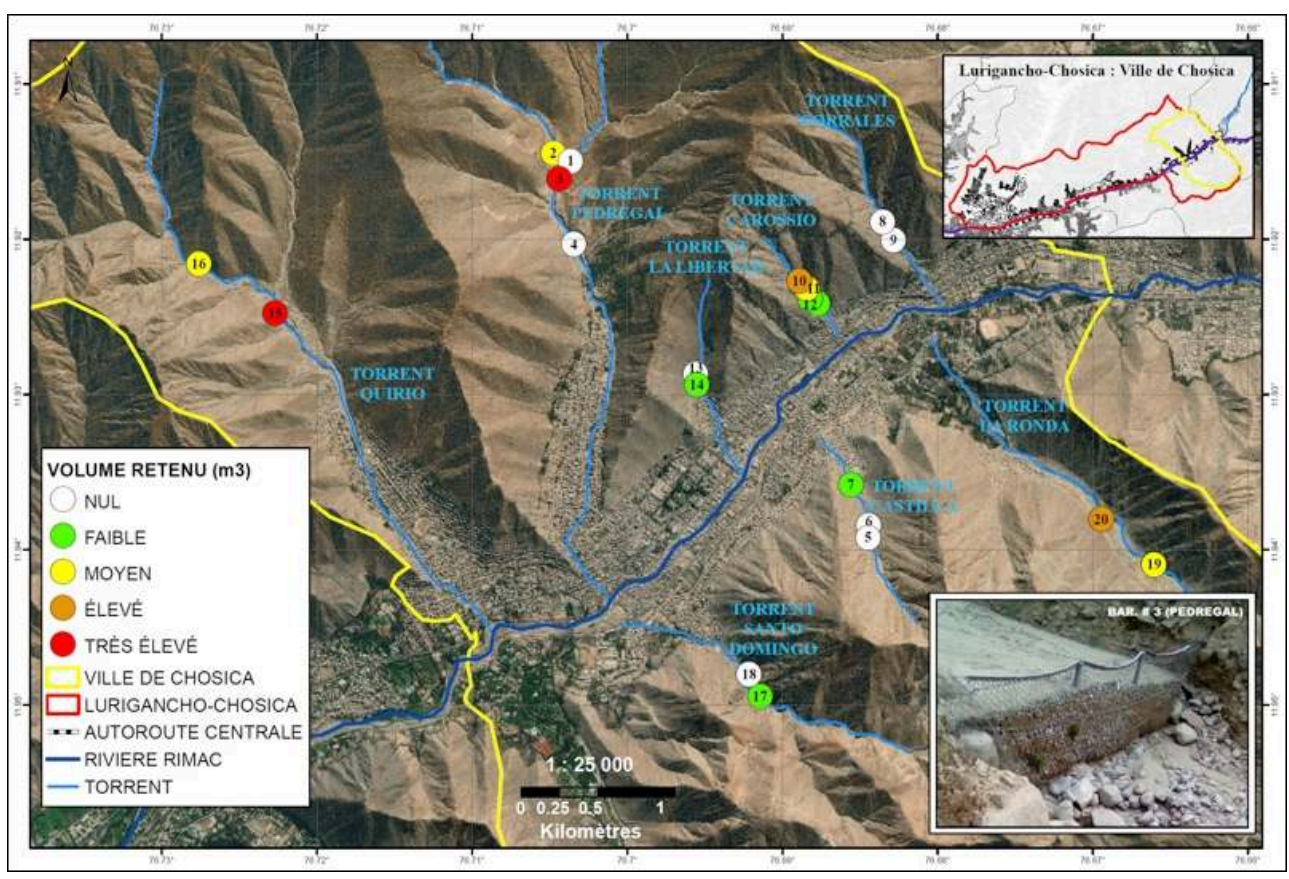

Sources: ANA, 2017 ; DigitalGlobe, 2016. Auteur : Alexis Mejía, juin 2018.

Finalement, si on compare l'information sur le matériel retenu et l'intensité des écoulements torrentiels par ravin (tableau 2), on peut clairement comprendre que la réduction de l'intensité des écoulements en 2017 est liée à la mise en place des barrières qui ont retenu une grande quantité de sédiments. S'il n'y avait pas eu de barrières, une grande partie de ces sédiments aurait été déplacée vers l'aval où se trouve la population, de telle sorte que dans le tableau 2 nous aurions enregistré davantage d'écoulements de très forte intensité en 2017. Cependant, des écoulements forts et très forts ont pu malgré tout se produire en lien avec la mobilisation de matériels sédimentaires issus des affluents en aval des barrières, et/ou de l'érosion du lit du ravin dans les tronçons à l'aval des barrières (principalement dans les bassins de grande taille). Cette supposition trouve des éléments de confirmation dans les témoignages d'habitants des ravins Pedregal et Quirio qui ont observé des apports sédimentaires des affluents latéraux ou provenant de l'érosion du fond du ravin.

Enfin, il apparait dans cette troisième partie sur la gestion des risques que malgré l'existence d'une politique nationale récente de gestion des risques, prédomine une gestion à l'échelle locale, au travers d'interventions ponctuelles, à l'initiative des résidents, d'ONG ou plus rarement des municipalités, avec l'appui éventuel de programmes nationaux.

\section{Conclusion}

59 Les écoulements torrentiels dans la banlieue de Lima (district de Lurigancho-Chosica) et leurs dommages (pertes de vie humaines, maisons détruites) ont été considérables en 1987 (Abad et al., 2009). Les derniers événements en 2012, 2015 et 2017 ont été moins meurtriers et sont mieux documentés (Nuñez et al., 2015 ; Guadalupe et Carillo 2012). Il 
a donc été possible d'analyser les différences entre les événements récents, en termes d'aléa, de fragilité de l'habitat et de nombre de personnes exposées aux écoulements torrentiels et finalement en termes de gestion du risque.

Les écoulements torrentiels ont été classés selon leur intensité et ont été mis en relation avec les précipitations. En effet, ne disposant pas d'informations sur les quantités de débris disponibles dans les bassins-versants et n'ayant pas pu effectuer ces mesures, nous avons considéré que la très grande quantité de débris disponible ne constituait pas un facteur limitant. Il apparaît que des pluies à Chosica sont presque quatre fois plus importantes que la normale $(120 \mathrm{~mm})$ en 2017, contre deux fois plus importantes en 2012 et 2015 (60 mm). De ce fait, elles ont donné lieu à des événements plus nombreux en 2017 mais pas toujours très intenses, selon l'indice que nous avons défini. De façon générale, l'indice d'intensité des écoulements torrentiels est très fortement corrélé aux pluies.

La vulnérabilité de la population exprimée par la fragilité des logements et l'exposition des personnes aux laves torrentielles évolue de façon contrastée. La fragilité des logements a diminué dans le temps puisque le pourcentage de logements en brique est passé de près de $60 \%$ dans les années 1980 et 1990 à $72 \%$ en 2007. Toutefois, le degré d'exposition à l'aléa a augmenté. En effet, le nombre de personnes vivant dans les zones " critiques ", où l'aléa est fort, des ravins Quirio, Pedregal, Carossio et Corrales, est passé de 27000 à 28000 entre 1993 et 2007, ce qui est considérable dans une ville de 58000 habitants.

La gestion du risque s'est enrichie de nouvelles mesures structurelles et non structurelles : mise en place d'un système d'alerte précoce, formation de la population en matière de gestion du risque, construction de nouvelles digues, et surtout installation de barrières flexibles en 2016. Les barrières flexibles ont probablement permis une diminution du niveau de risque puisqu'en 2017, elles ont retenu de grandes quantités de matériel sédimentaire en amont, réduisant ainsi l'intensité de presque tous les écoulements torrentiels vers aval, là où se localise la population. Même si l'aléa de 2017 a été plus fort que les précédents, l'intensité des événements et les dommages n'ont pas été des plus élevés.

Cependant, les dégâts de 2017 montrent que cette mesure ne semble pas suffisante car la présence de logements dans des zones d'aléa fort reste un facteur de risque. Des études sociales approfondies sur l'exposition des populations et leurs causes seraient pour cette raison très utiles, de même que des travaux sur les mesures de prévention. Manquent aussi des études plus fouillées sur les écoulements torrentiels afin d'améliorer les ouvrages mis en place. Ceci implique l'analyse quantitative et qualitative du matériel sédimentaire disponible pour le transport, sujet qui n'a été abordé dans cette recherche. Il serait utile d'étudier le matériel retenu par les barrières flexibles: analyses granulométriques, analyses de la trajectoire et de l'origine du matériel (ravin principal, affluent, lit), comparaisons entre les bassins versants torrentiels pour mieux comprendre la géomorphologie de chacun. Ces études pourraient être réalisées dans un travail ultérieur avec des mesures sur le terrain, en utilisant les images de drones, ainsi qu'en définissant plus précisément l'intensité des flux en installant des caméras dans les bassins versants torrentiels. Ainsi, les actions pour faire face aux écoulements torrentiels doivent être assumées par la communauté scientifique, en plus de l'être par la population elle-même et par les autorités. 


\section{BIBLIOGRAPHIE}

Abad C., 2009. Huaycos en 1987 en el distrito de Lurigancho-Chosica. Bulletin de l'Institut Français d'Etudes Andines, vol. 38, n 3, p. 475-486.

ANA (Autorité Nationale de l'Eau du Pérou), 2010. Evaluación de los Recursos Hídricos en la Cuenca del Río Rímac. Lima, Ministère de l'Agriculture du Pérou - MINAGRI, 225 p.

ANA (Autorité Nationale de l'Eau du Pérou), 2015a. Actividades de limpieza y protección en quebradas por peligro inminente de inundaciones y huaycos en los departamentos de Lima, Piura, Tumbes, Lambayeque, La Libertad, Cajamarca y Cusco. Lima, Ministère de l'Agriculture du Pérou - MINAGRI, $263 \mathrm{p}$.

ANA (Autorité Nationale de l'Eau du Pérou), 2015b. Contrato de suministro, instalación y puesta en operación de 22 barreras dinámicas contra flujo de detritos ). Lima, Ministère de l'Agriculture du Pérou - MINAGRI, $9 \mathrm{p}$.

ANA (Autorité Nationale de l'Eau du Pérou), 2016. ANA está culminando instalación de 22 barreras dinámicas en Lurigancho-Chosica ). http://www.ana.gob.pe/video/ana-esta-culminandoinstalacion-de-22-barreras-dinamicas-en-lurigancho-chosica. Consulté le 18 avril 2018.

ANA (Autorité Nationale de l'Eau du Pérou), 2017. Descolmatación y conservación de barreras dinámicas contra el flujo de detritos. Lima, $21 \mathrm{p}$.

ANA (Autorité Nationale de l'Eau du Pérou), 2018a. Calendario de los trabajos de limpieza de las barreras dinámicas 2018. Information du bureau de l'unité exécutive $\mathrm{n}^{\circ} 2 \mathrm{du}$ projet Modernisation de la Gestion des Ressources Hydriques de l'ANA. Lima, 23 p.

ANA (Autorité Nationale de l'Eau du Pérou), 2018b. Enregistrement photographique des travaux de nettoyage et d'entretien des barrières flexibles.

BID (Banque interaméricaine de développement), Centre de l'eau pour l'Amérique latine et les Caraibes, Institut de technologie de Monterrey, 2015. Agua y ciudades en América Latina: Retos para el desarrollo sostenible. DOI : http://dx.doi.org/10.18235/0001107

CENEPRED (Centre National d'Estimation, Prévention et Réduction du Risque de Catastrophes du Pérou), 2018. Glosario de Términos. Lima, 6 p.

CENAPRED (Centre National de Prévention de Catastrophes du Mexique), 2001. Diagnóstico de Peligros e Identificación de Riesgos de Desastres en México. Mexico, 261 p.

COEN-INDECI (Centre d'Opérations de Secours National du Pérou de l'Institut National de Défense Civile du Pérou), 2015. Huaycos afectan el distrito de Lurigancho Chosica - Lima Metropolitana. Rapport Technique $n^{\circ}$ 581-08/05/2015/COEN-INDECI. Lima, Institut National de Défense Civile du Pérou INDECI, $29 \mathrm{p}$.

COE-CHOSICA (Centre d'opérations de Secours du district de Lurigancho-Chosica), 2017. Base de données statistiques, photographiques et audiovisuelles.

D'Ercole R., Chandes J., Perfettini H., Audin L., 2007. Le séisme de Pisco du 15 août 2007 : entre urgence et reconstruction. EchoGéo [En ligne], Rubrique Sur le Vif. URL : http:// journals.openedition.org/echogeo/2109; DOI : 10.4000/echogeo.2109.

D’Ercole R., Hardy S., Metzger P. et Robert J., 2009. Vulnérabilités urbaines dans les pays andins. Introduction générale. Bulletin de l'Institut français d'études andines [En ligne], vol. 38, n 3, p. 411-420. http://journals.openedition.org/bifea/2227 ; DOI : 10.4000/bifea.2227 
DIPECHO (Programme de Préparation aux Catastrophes d'ECHO), 2016. Informe Final: Línea de base comunitaria de las quebradas Carosio y Mariscal Castilla del distrito de Lurigancho-Chosica. Lima, $47 \mathrm{p}$.

DNU (Direction Nationale d'Urbanisme au Pérou), 2011. Reglamento de Acondicionamiento Territorial y Desarrollo Urbano. Lima, Ministère du Logement, de la Construction et de l'Assainissement du Pérou - MVCS, 29 p.

ENFEN (Étude nationale du phénomène El Niño au Pérou), 2017. Niño Costero 2017. Rapport Technique extraordinaire $\mathrm{n}^{\circ}$ 001-2017/ENFEN. Lima, $31 \mathrm{p}$.

García A., 2007. Medidas estructurales y medidas no estructurales de defensa frente a inundaciones. Madrid, École d'Organisation Industriel, 22 p.

Guadalupe E., Carrillo N., 2012. Caracterización y análisis de los huaycos del 5 de abril del 2012 ChosicaLima. Revue de l'Institut de Recherche de la Faculté d'Ingénierie Géologique, Minière, Métallurgique et Géographique de l'Université Nationale de San Marcos, vol. 15, n² 29, p. 69-82.

Hungr O., Evans S. G., Bovis M. J., Hutchinson J. N, 2001. A review of the classification of landslides of the flow type. Environmental and Engineering Geoscience, vol. 7, p. 221-238.

Huebl, J., Fiebiger, G., 2005. Debris flow mitigation measures. In Jakob M., Hungr O., Debris Flow Hazard and Related Phenomena. Heidelberg, Springer, p. 445-485.

INDECI (Institut National de Défense Civile du Pérou), 2005. Mapa de peligros y plan de usos del suelo y medidas de mitigación ante desastres de la ciudad de Chosica. Lima, Projet INDECI-PNUD - Villes Durables, 48 p.

INEI (Institut National de Statistique et d'informatique du Pérou), 1981. Censos Nacionales 1981: VIII de Población y III de Vivienda. Base de données statistique. http://censos.inei.gob.pe/censos1981/ redatam/ (consultée le 10 avril 2017).

INEI (Institut National de Statistique et d'informatique du Pérou), 1993. Censos Nacionales 1993: IX de Población y IV de Vivienda. Base de données statistique. http://censos.inei.gob.pe/censos1993/ redatam/. Consultée le 10 avril 2017 au Ministère du Logement.

INEI (Institut National de Statistique et d'informatique du Pérou), 2007. Censos Nacionales 2007: XI de Población y VI de Vivienda. Base de données statistique et géographique - GIS.

Lavado W., Ronchail J., Labat D., Espinoza J., Guyot J., 2012. Basin-scale analysis of rainfall and runoff in Peru (1969-2004): Pacific, Titicaca and Amazonas drainages. Hydrological Sciences Journal [En ligne], vol. 57, n 4, p. 625-642, DOI : 10.1080/02626667.2012.672985

Mairie de Lurigancho-Chosica, 2013. Plan de Desarrollo Concertado Distrital de Lurigancho Chosica 2013-2025. Lima, 116 p.

Mairie de Lurigancho-Chosica, 2016. Gestión de riesgo de desastres 2016 [Diapositives PowerPoint]. Centre d'Opérations de Secours du district de Lurigancho-Chosica - COE-CHOSICA. Lima, exposé de mars 2016.

Meunier M., 1991. Éléments d'Hydraulique Torrentielle. Éditions Cemagref, Antony, 278 p.

Metzger P., D’Ercole, 2011. Les risques en milieu urbain. Echogéo [En ligne], nº 18. http:// journals.openedition.org/echogeo/12640 - DOI : 10.4000/echogeo.12640

Nuñez S., Villacorta S., Huarez C., 2015. Evaluación geodinámica de los flujos de detritos del 23/03/2015 entre las quebradas Rayos de Sol y Quirio (Chosica) y Cashahuacra (Santa Eulalia). Rapport Technique $\mathrm{n}^{\circ} \mathrm{A}$-6680. Lima, Institut Géologique, Minier et Métallurgique du Pérou - INGEMMET, 65 p. 
Perrin J. L., Fourcade B., Poulenard J., Richard D., Sierra A. 2000. Quito face à un risque d'origine naturelle : les laves torrentielles. Revue de géographie alpine, vol. 88, p. 43-57.

Peru 21, le 23 janvier 2013. Chosica: Reforzarán diques para proteger viviendas de posible huaico [En ligne]. https://peru21.pe/lima/chosica-reforzaran-diques-proteger-viviendas-posiblehuaico-86688-noticia/ (consulté le 22 mars 2018).

Pigeon P., 2003. L'intérêt du risque pour l'enseignement de la géographie. In Moriniaux V., Les risques. Paris, Editions du temps, p. 9-21.

PREDES (Centre d'études et de prévention de catastrophes), 2015. Huaycos en Chosica. Rapport Spécial. Lima, $19 \mathrm{p}$.

Rodriguez-Morata C., Ballesteros-Cánovas J. A., Rohrer M., Espinoza J. C., Beniston M., Stoffel M., 2018. Linking atmospheric circulation patterns with hydro-geomorphic disasters in Peru. International Journal of Climatology, vol. 38, p. 3388-3404.

Rodríguez-Morata C., Villacorta S., Stoffel M., Ballesteros-Cánovas J. C., 2019. Assessing strategies to mitigate debris-flow risk in Abancay province, South-Central Peruvian Andes. Geomorphology, vol. 342, p. 127-139.

Sierra A., Robert J., Durand M. et Abad C. 2009. Experiencias de gestión de riesgos en Lima: actores y territorios urbanos. Bulletin de l'Institut français d'études andines [En ligne], vol. 38, $\mathrm{n}^{\circ} 3$. http://journals.openedition.org/bifea/2433 - DOI : 10.4000/bifea.2433

Schumm S., 1973. Geomorphic Thresholds and Complex Response of Drainage Systems. In Morisawa M. (ed.), Fluvial Geomorphology. Publications of Geomorphology, State University of New York, Binghamton, 299-310.

SENAMHI (Service National de Météorologie et d'Hydrologie du Pérou), 2018a. Base de données des enregistrements de pluie journalière des stations météorologiques du SENAMHI localisées dans le bassin versant de la rivière Rímac entre 2012 et 2017.

SENAMHI (Service National de Météorologie et d'Hydrologie du Pérou), 2018b. Base de données PISCO-SENAMHI, 1981-2017.

Takahashi K., 2017. Fenómeno El Niño: “Global” vs “Costero”. Boletín Técnico El Niño, vol. 4, n 4, p. 4-7.

Thouret J. C., D'Ercole R. 1994. Les phénomènes naturels créateurs de dommages (= menaces) : diagnostic, inventaire et typologie. Revue de géographie alpine, vol. 82, p. 17-25.

Tumialán P., 2015. Rasgos geológicos del río Rímac, abastecimiento de agua en su cono de deyección. Revista de Ciencias, vol. 11, p. 120-130.

Zambrano E., 1986. El fenómeno de El Niño y la Oscilación Sur (ENSO). Acte Océanographique du Pacifique, vol. 3, n 1, p. 195-203.

\section{NOTES}

1. $2012: 2$ décès, 81 logements détruits (SINPAD-INDECI, consulté le 22 mars 2018) ; 2015 : 9 décès, 107 logements détruits (COEN-INDECI, 2015) ; 2017 : aucun décès, 100 logements détruits (SINPAD-INDECI, consulté le 22 mars 2018).

2. Institutions : Université Nationale de San Marcos - UNMSM (Guadalupe et Carrillo, 2012) ; ONG PREDES (PREDES, 2015); Institut Géologique, Minier et Métallurgique du Pérou - INGEMMET 
(Nuñez et al., 2015); Institut National de Défense Civile du Pérou - INDECI (SINPAD-INDECI, consulté le 22 mars 2018).

3. La quantité de pluie en 2017 a été supérieure à celle des années précédentes principalement à cause de l'arrivée du phénomène océano-atmosphérique appelé El Niño Costero, qui a favorisé l'arrivée d'eaux chaudes du Pacifique équatorial. Celles-ci font augmenter rapidement la température de surface océanique (TSO) de la côte péruvienne et de l'Equateur en très peu de temps et de manière inattendue (Takahashi, 2017) (par exemple, sur la côte de La Libertad, au nord du Pérou, la TSO est passée de $17{ }^{\circ} \mathrm{C}$ à $27^{\circ} \mathrm{C}$ en deux semaines vers mi-janvier 2017, cf. ENFEN, 2017), ce qui est exceptionnel, et favorisant de fortes pluies par convection. Même si des événements El Niño se produisent en 2012 et 2015, en lien avec des eaux plus chaudes que la normale à l'Est du Pacifique, les affaiblissements résultant de l'anticyclone du Pacifique Sud n'ont pas eu un impact aussi important sur les pluies à Chosica qu'en 2017. Enfin il convient de préciser que les désastres géomorphologiques au Pérou ne sont pas toujours liés à El Niño (RodriguezMorata et al., 2018).

4. L'augmentation de la population a probablement continué depuis 2007 compte tenu du taux de croissance antérieur (+ 3,8 \% entre 1993 et 2007) et du développement économique dans la région. Cependant, ces données sont utilisées car ce sont les seules données officielles disponibles au moment de l'élaboration du document.

5. Le terme « invasion » fait référence à l'occupation arbitraire d'un lieu, sans en avoir le droit, parfois de façon violente. Les invasions correspondent à une expansion urbaine non contrôlée par les pouvoirs publics, produite par une forte croissance démographique (BID, 2015). Habituellement, les invasions ont lieu dans des terrains vagues, où les occupants attentent que l'État leur fournissent les services basiques (service de l'eau et de l'assainissement, ainsi que l'électricité), sous la justification (qui est vraie) que les logements manquent face à une demande importante.

6. Communication personnelle de Monsieur José Martinez, chef du COE-CHOSICA, le 22 février 2017.

7. Ibid.

8. ECHO, European Commission's Humanitarian Aid and Civil Protection Department (traduit par "Commission européenne à l'aide humanitaire et à la protection civile »).

9. KOICA, Korea International Cooperation Agency (traduit par « Agence Coréenne de Coopération Internationale »)

10. Communication personnelle de Monsieur José Martinez, chef du COE-CHOSICA, le 23 février 2018.

11. Communication personnelle de Monsieur Juan Gomez, directeur de la sous-direction de Sciences de la Terre Solide de l'Institut de Géophysique du Pérou - IGP, le 20 février 2018.

12. Les leaders de quartiers rencontrés sont notamment Madame Victoria Prudencio du quartier San Antonio (2ème Zone, ravin Pedregal) et Monsieur Orlando Jimenez, du quartier Nicolás del Piérola (ravin Quirio).

13. Communication personnelle de Monsieur José Martinez, chef du COE-CHOSICA, le 22 février 2017.

14. Barreras Dinámicas (Barrières Dynamiques, en espagnol) est le nom utilisé dans le cadre du projet qui a concerné l'installation de ces structures à Chosica en 2016, mais son nom technique en français est « Barrières Flexibles » (site Geobrugg, consulté le 18 avril 2018).

15. Le Nuevo Sol (S/.) est la monnaie nationale du Pérou ; 1 US\$ = S/. 3,2231, le 16 avril 2018.

16. Communication personnelle de Monsieur Felix Caubi, contrôleur général des travaux de nettoyage des Barrières Flexibles de l'ANA, le 19 février 2018.

17. Communication personnelle de Monsieur Felix Caubi, contrôleur général des travaux de nettoyage des Barrières Flexibles de l'ANA, le 19 février 2018. 


\section{RÉSUMÉS}

La ville de Chosica (district de Lurigancho-Chosica, dans la banlieue de Lima au Pérou), à cause de ses caractéristiques physiques, est fréquemment frappée par des écoulements torrentiels. Ces événements ne sont pas seulement liés à l'occurrence de pluies fortes (elles-mêmes le plus souvent liées au phénomène d'El Niño), mais aussi aux conditions d'instabilité géomorphologique dans des ravins, dont les parties inférieure et centrale sont peuplées. Ces événements produisent de fortes pertes de vies humaines et économiques. Nous analysons l'aléa (l'intensité et la fréquence des écoulements torrentiels), la fragilité des logements, le degré d'exposition face à l'aléa, et le niveau de gestion du risque lors des trois derniers événements de 2012, 2015 et 2017. L'installation de barrières flexibles en 2016 semble avoir contribué à la réduction des dégâts en 2017, alors que les pluies ont été plus fortes que les années précédentes.

Chosica city (capital of Lurigancho-Chosica district, in Lima city in Peru), because of its physical characteristics, is affected frequently by torrential flows. These events are not only related to heavy rainfalls (usually when the El Niño phenomenon happens), but also because of the geomorphologic conditions of the torrents, which are colonized in their low and central parts, producing human losses and economic damages. We analyze the hazard (the intensity and frequency of torrential flows), the fragility of housing, the degree of exposure to the hazard, and the level of risk management during the last three events of 2012, 2015 and 2017. The installation of flexible barriers in 2016 appears to have contributed to damage reduction in 2017, when rains were heavier than in previous years.

La ciudad de Chosica (capital del distrito de Lurigancho-Chosica, en la ciudad de Lima en el Perú), a causa de sus características físicas, es afectada frecuentemente por los flujos torrenciales. Estos eventos no están asociados solamente a lluvias intensas (normalmente cuando ocurre el fenómeno El Niño), sino también a las condiciones de inestabilidad geomorfológica de las quebradas, cuyas partes inferior y central están pobladas, produciendo grandes pérdidas de vidas humanas y económicas. Analizamos el peligro (la intensidad y frecuencia de los flujos torrenciales), la fragilidad de las viviendas, el grado de exposición al peligro y el nivel de gestión del riesgo durante los tres últimos eventos de 2012, 2015 y 2017. La instalación de barreras flexibles en 2016 parece haber contribuido a reducir los daños en 2017, cuando las lluvias eran más intensas que en años anteriores.

\section{INDEX}

Mots-clés : Chosica (Pérou), écoulement torrentiel, fragilité, degré d'exposition, gestion du risque de catastrophe

Palabras claves : Chosica (Perú), flujo torrencial, fragilidad, grado de exposición, gestión del riesgo de desastre

Keywords : Chosica (Peru), torrential flow, fragility, degree of exposure, disaster risk management 


\section{AUTEURS}

\section{ALEXIS RICARDO MEJÍA UQUICHE}

Alexis Ricardo Mejía Uquiche, alexismeju@hotmail.com, est analyste de projets à la Direction de l'Exécution de Programmes et Projets en Construction et Assainissement du Ministère de Logement (Lima, Pérou).

\section{JOSYANE RONCHAIL}

Josyane Ronchail, josyaneronchail@locean-ipsl.upmc.fr, est maître de conférences à l'université Paris Diderot et memebre du Laboratoire d'Océanographie et du Climat (LOCEAN). Elle a récemment publié :

- Ronchail J., Espinoza J. C., Drapeau G., et al., 2018. The flood recession period in Western Amazonia and its variability during the 1985-2015 period. Journal of Hydrology: Regional Studies [En ligne], vol. 15, p. 16-30. DOI : 10.1016/j.ejrh.2017.11.008

Espinoza J. C., Ronchail J., Marengo JA., Segura H. 2018. Contrasting North-South changes in Amazon wet-day and dry-day frequency and related atmospheric features (1981-2017). Climate Dynamics [En ligne], $\mathrm{n}^{\circ}$ 52, p. 5413-5430. DOI : 10.1007/s00382-018-4462-2 